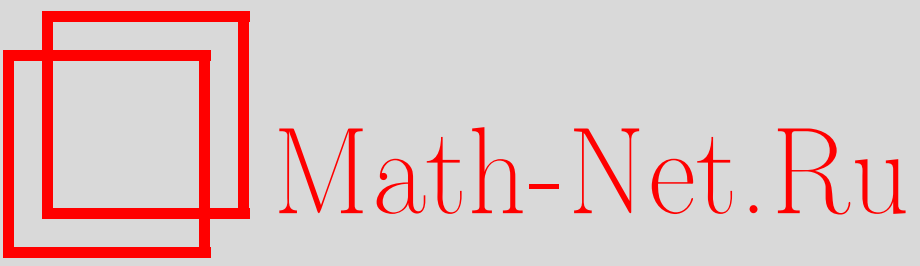

А. А. Кузнецова, А. С. Холево, Теоремы кодирования для гибридных каналов, Теория вероятн. и ее примен., 2013, том 58, выпуск 2, 298-324

DOI: https://doi.org/10.4213/tvp4508

Использование Общероссийского математического портала Math-Net.Ru подразумевает, что вы прочитали и согласны с пользовательским соглашением

http://www.mathnet.ru/rus/agreement

Параметры загрузки:

IP: 35.174 .16 .151

26 апреля 2023 г., 16:07:36 


\title{
ТЕОРЕМЫ КОДИРОВАНИЯ ДЛЯ ГИБРИДНЫХ КАНАЛОВ ${ }^{1)}$
}

\begin{abstract}
Основным результатом настоящей работы является теорема кодирования для пропускной способности с использованием сцепленного состояния (entanglement-assisted capacity) в случае измерительного квантового канала связи с произвольным алфавитом (теорема 3). Попутно получен ряд результатов для энтропий гибридных (классически-квантовых) систем, а также для пропускных способностей канала с гибридным выходом, которые используются при доказательстве теоремы 3.
\end{abstract}

Ключевые слова и фразы: канал связи, гибридная (классическиквантовая) вероятностная система, классическая пропускная способность, сцепленное состояние, теорема кодирования.

1. Введение. В последние два десятилетия значительное развитие получило квантовое обобщение теории информации, в котором ключевую роль играет понятие сцепленности (entanglement) - специфической квантовой корреляции между компонентами составной квантовой системы (см., например, [1], [2]). В пионерской работе П. Шора с соавторами [3] был рассмотрен протокол передачи информации по квантовому каналу связи с использованием сцепленности между входом и выходом канала. В случае конечномерных каналов была доказана теорема кодирования, дающая явное выражение для соответствующей пропускной способности через максимум квантовой взаимной информации по всевозможным входным состояниям (в настоящей работе речь всюду идет о классических пропускных способностях, т.е. о передаче классических данных через квантовый канал связи). Сравнение этого выражения с обычной пропускной способностью показывает возможность многократного увеличения скорости передачи для квантовых каналов с шумом благодаря использованию сцепленности. В частности, подобный выигрыш для так называемых измерительных каналов продемонстрирован в работе [4], где рассмотрен ряд конкретных примеров, представляющих

* МГТУ ИМ. Н.Э. Баумана, Москва, Россия; e-mail: kuznetsova.a.a@bk.ru

** Математический институт им. В.А. Стеклова РАН, Москва, Россия; е-mail: holevo@mi.ras.ru

1) Работа выполнена при поддержке РФФИ (грант № 12-01-00319) и Российского Квантового Центра. 
интерес для приложений (квантовая томография в конечномерном пространстве, оптическое гетеродинирование с ограничением на энергию входного сигнала и др.). При этом в случае измерений с дискретным алфавитом (множеством значений) $\mathscr{Y}$ измерительный канал $\mathscr{M}$ можно рассматривать как квантовый, и к нему применимы результаты работы [3] в случае конечного $\mathscr{Y}$, либо их бесконечномерное обобщение [5] в случае счетного $\mathscr{Y}$. Для измерений же с непрерывным алфавитом $\mathscr{Y}$ подобный подход оказывается невозможным и формулировка протокола передачи информации с использованием сцепленности, а также доказательство соответствующей теоремы кодирования требуют рассмотрения так называемых гибридных (классически-квантовых) вероятностных систем.

Основным результатом настоящей работы является теорема кодирования для пропускной способности с использованием сцепленного состояния (entanglement-assisted capacity) в случае измерительного канала с произвольным измеримым алфавитом (теорема 3). Тем самым получает обоснование формула (28), анонсированная в работе [4]. Попутно мы получаем ряд результатов для энтропий гибридных систем, а также для пропускных способностей каналов с гибридным выходом, которые используются при доказательстве теоремы 3. Более подробные объяснения используемых в работе понятий квантовой теории информации, а также обозначений (в частности, бра- и кет-векторов Дирака) можно найти, например, в книгах [1], [2].

2. Некоторые сведения о гибридных системах. Квантовая система описывается сепарабельным гильбертовым пространством $\mathscr{H}$. Далее $\mathfrak{B}(\mathscr{H})$ - алгебра всех ограниченных операторов в $\mathscr{H}, \mathfrak{T}(\mathscr{H})-$ пространство операторов со следом (ядерных операторов), $\mathfrak{S}(\mathscr{H})-$ выпуклое множество операторов плотности (положительных операторов с единичным следом, называемых также квантовыми состояниями) в $\mathscr{H}[6]$. Введем также пространство с мерой $(\Omega, \mathscr{F}, \mu)$, где $\Omega-$ некоторое множество, $\mathscr{F}-\sigma$-алгебра его подмножеств, $\mu-\sigma$-конечная мера на $\mathscr{F}$.

Гибридная (классически-квантовая, кратко сq-) система описывается алгеброй фон Неймана $\mathscr{L}=\mathscr{L}^{\infty}(\Omega, \mathscr{F}, \mu, \mathfrak{B}(\mathscr{H}))$, элементами которой являются слабо измеримые существенно ограниченные функции $X(\omega), \omega \in \Omega$, со значениями в $\mathfrak{B}(\mathscr{H})$. Рассмотрим также предсопряженное пространство $\mathscr{L}_{*}=\mathscr{L}_{1}(\Omega, \mathscr{F}, \mu, \mathfrak{T}(\mathscr{H}))$, элементами которого являются измеримые функции $S=\{S(\omega)\}$ со значениями в $\mathfrak{T}(\mathscr{H})$, интегрируемые по мере $\mu$ (см. подробнее [7], [8]).

О п р е д е л е н и е 1 . Состоянием (сq-состоянием) на алгебре $\mathscr{L}$ называется элемент $S=\{S(\omega)\} \in \mathscr{L}_{*}$ такой, что

$$
S(\omega) \geqslant 0 \quad(\bmod \mu), \quad \int_{\Omega} \operatorname{Tr} S(\omega) \mu(d \omega)=1 .
$$


Частичным q-состоянием называется оператор плотности

$$
S^{\mathrm{q}}=\int_{\Omega} S(\omega) \mu(d \omega)
$$

частичным с-состоянием называется вероятностная мера $S^{\text {c }}$ на $(\Omega, \mathscr{F}, \mu)$, определяемая плотностью $\operatorname{Tr} S(\omega)$.

Будем обозначать $\mathfrak{S}(\Omega, \mathscr{H})$ выпуклое множество всех сq-состояний.

Следуя [8], введем понятия энтропии и относительной энтропии сqсостояний. Здесь и далее мы используем обозначения "c, q, сq" для энтропийных характеристик классических, квантовых и гибридных систем соответственно. По поводу определений и свойств квантовых энтропий см., например, [2].

О п р е д е л е н и е 2 . Энтропия сq-состояния $S$ определяется соотношением

$$
H_{\mathrm{cq}}(S)=\int_{\Omega} H_{\mathrm{q}}(S(\omega)) \mu(d \omega),
$$

где $H_{\mathrm{q}}(S)=-\operatorname{Tr} S \log S$ - энтропия фон Неймана положительного опеpaтора $S \in \mathfrak{T}(\mathscr{H})$.

Заметим, что

$$
H_{\mathrm{cq}}(S)=H_{\mathrm{c}}(\Omega)+\int_{\Omega} p(\omega) H_{\mathrm{q}}(\widehat{S}(\omega)) \mu(d \omega),
$$

где $p(\omega)=\operatorname{Tr} S(\omega), \widehat{S}(\omega)=(p(\omega))^{-1} S(\omega), H_{\mathrm{c}}(\Omega)$ - дифференциальная энтропия распределения вероятностей с плотностью $p(\omega)$ относительно меры $\mu$.

О п р е д е л е н и е 3 . Относительная энтропия сq-состояний $S_{1}$, $S_{2}$ определяется соотношением

$$
H_{\mathrm{cq}}\left(S_{1} \| S_{2}\right)=\int_{\Omega} H_{\mathrm{q}}\left(S_{1}(\omega) \| S_{2}(\omega)\right) \mu(d \omega),
$$

где

$$
H_{\mathrm{q}}\left(S_{1}(\omega) \| S_{2}(\omega)\right)=\operatorname{Tr} S_{1}(\omega)\left(\log S_{1}(\omega)-\log S_{2}(\omega)\right)
$$

— квантовая относительная энтропия.

Введем также понятие, которое будет играть важную роль в настоящей статье.

О п р ед е л е н и е 4. Пусть $S$ - сq-состояние, такое что $H_{\mathrm{q}}\left(S^{q}\right)<\infty$. Условной энтропией сq-системы $\Omega \mathscr{H}$ относительно qсистемы $\mathscr{H}$ называется величина

$$
H_{\mathrm{cq}}(\Omega \mid \mathscr{H})=H_{\mathrm{cq}}(S)-H_{\mathrm{q}}\left(S^{\mathrm{q}}\right) .
$$


Заметим, что определение 4 есть аналог определения квантовой условной энтропии в случае конечномерных гильбертовых пространств (об определении квантовой условной энтропии в бесконечномерном случае см. [9]).

Bce определенные выше энтропии могут принимать значения в $(-\infty,+\infty]$

Далее мы рассматриваем различные квантовые системы $A, B, \ldots$, которые описываются гильбертовыми пространствами $\mathscr{H}_{A}, \mathscr{H}_{B}, \ldots$ Энтропию сq-состояния $S \in \mathfrak{S}\left(\Omega, \mathscr{H}_{A}\right)$ будем кратко обозначать $H_{\text {сq }}(\Omega A)$ и т.п. Составная квантовая система описывается тензорным произведением соответствующих гильбертовых пространств $\mathscr{H}_{A} \otimes \mathscr{H}_{B}$ (см. подробнее [6], [2]).

Лемма 1. Энтропия сq-системь обладает следуюшими свойствами.

1. Субаддитивность-1:

$$
H_{\mathrm{c}}(\Omega) \leqslant H_{\mathrm{cq}}(\Omega A) \leqslant H_{\mathrm{c}}(\Omega)+H_{\mathrm{q}}(A) .
$$

2. Субаддитивность-2:

$$
H_{\mathrm{cq}}(\Omega A B) \leqslant H_{\mathrm{cq}}(\Omega A)+H_{\mathrm{cq}}(\Omega B) .
$$

3. Сильная субаддитивность:

$$
H_{\mathrm{cq}}(\Omega A B)+H_{\mathrm{q}}(A) \leqslant H_{\mathrm{cq}}(\Omega A)+H_{\mathrm{q}}(A B) .
$$

Д о к а з а т е л ь с т в о. 1. Неравенство

$$
H_{\mathrm{cq}}(\Omega A) \geqslant H_{\mathrm{c}}(\Omega)
$$

очевидно выполняется для любой системы $\Omega A$ по определению энтропии гибридной системы. Для доказательства второго неравенства обозначим $p(\omega)=\operatorname{Tr} S_{A}(\omega), \widehat{S}_{A}(\omega)=p(\omega)^{-1} S_{A}(\omega)$. В этих обозначениях $H_{\mathrm{c}}(\Omega)-$ классическая дифференциальная энтропия распределения вероятностей с плотностью $p(\omega)$ относительно меры $\mu$. Тогда, используя вогнутость энтропии фон Неймана, получаем

$$
H_{\mathrm{cq}}(\Omega A)=H_{\mathrm{c}}(\Omega)+\int_{\Omega} p(\omega) H_{\mathrm{q}}\left(\widehat{S}_{A}(\omega)\right) \mu(d \omega) \leqslant H_{\mathrm{c}}(\Omega)+H_{\mathrm{q}}\left(S_{A}^{\mathrm{q}}\right) .
$$

2. Если $H_{\mathrm{cq}}(\Omega A)+H_{\mathrm{cq}}(\Omega B)<\infty$, то неравенство следует из неотрицательности относительной энтропии для гибридных систем. В противном случае неравенство очевидно.

3. Если $H_{\mathrm{q}}(A B)=\infty$, то неравенство (4) очевидно выполняется. Если $H_{\mathrm{q}}(A)=\infty$, то, воспользовавшись фактом, что $H_{\mathrm{cq}}(\Omega A) \geqslant H_{\mathrm{q}}(A)$, 
снова получаем выполнение неравенства. Если же $H_{\mathrm{c}}(\Omega)=\infty$, то неравенство (4) следует из (5).

Теперь рассмотрим случай $H_{\mathrm{c}}(\Omega)<\infty, H_{\mathrm{q}}(A)<\infty, H_{\mathrm{q}}(A B)<\infty$. Заметив, что $p(\omega)=\operatorname{Tr} S_{A B}(\omega)=\operatorname{Tr} S_{A}(\omega)$, и обозначив

$$
\widehat{S}_{A B}(\omega)=p(\omega)^{-1} S_{A B}(\omega), \quad \widehat{S}_{A}(\omega)=p(\omega)^{-1} S_{A}(\omega),
$$

перепишем искомое неравенство в виде

$$
H_{\mathrm{cq}}(\Omega A B)-H_{\mathrm{q}}(A B) \leqslant H_{\mathrm{cq}}(\Omega A)-H_{\mathrm{q}}(A),
$$

что равносильно

$$
\begin{aligned}
& H_{\mathrm{c}}(\Omega)-\int_{\Omega} p(\omega) H_{\mathrm{q}}\left(\widehat{S}_{A B}(\omega) \| S_{A B}^{\mathrm{q}}\right) \mu(d \omega) \\
& \quad \leqslant H_{\mathrm{c}}(\Omega)-\int_{\Omega} p(\omega) H_{\mathrm{q}}\left(\widehat{S}_{A}(\omega) \| S_{A}^{\mathrm{q}}\right) \mu(d \omega),
\end{aligned}
$$

а последнее неравенство следует из монотонности квантовой относительной энтропии. Лемма 1 доказана.

Из сильной субаддитивности энтропии для гибридных систем непосредственно следует свойство монотонности условной энтропии.

Лемма 2. Рассмотрим гильбертовь пространства $\mathscr{H}_{A}, \mathscr{H}_{B}$ и состояния

$$
S_{A} \in \mathfrak{S}\left(\Omega, \mathscr{H}_{A}\right), \quad S_{A}^{\mathrm{q}}=\int_{\Omega} S_{A}(\omega) \mu(d \omega) \in \mathfrak{S}\left(\mathscr{H}_{A}\right),
$$

а также

$$
S_{A B} \in \mathfrak{S}\left(\Omega, \mathscr{H}_{A} \otimes \mathscr{H}_{B}\right), \quad S_{A B}^{\mathrm{q}}=\int_{\Omega} S_{A B}(\omega) \mu(d \omega) \in \mathfrak{S}\left(\mathscr{H}_{A} \otimes \mathscr{H}_{B}\right),
$$

удовлетворяюшие условиям $H\left(S_{A}^{q}\right)<+\infty, H\left(S_{A B}^{\mathrm{q}}\right)<+\infty$, причем для почти всех $\omega$ выполняется равенство

$$
\operatorname{Tr}_{B} S_{A B}(\omega)=S_{A}(\omega) .
$$

Тогда

$$
H_{\mathrm{cq}}(\Omega \mid A B) \leqslant H_{\mathrm{cq}}(\Omega \mid A) .
$$

Далее нам также понадобится понятие наблюдаемой в гибридной системе.

О п р е д е л е н и е 5 . Пусть Я - конечное множество. Наблюдаемой $M$ со значениями в $\mathscr{Y}$ в гибридной системе называется разложение единицы в $\mathscr{L}$, т.е. набор элементов $\left\{M_{y} ; y \in \mathscr{Y}\right\} \subset \mathscr{L}$, где $M_{y}=\left\{M_{y}(\omega)\right\}$, таких, что

$$
M_{y}(\omega) \geqslant 0(\bmod \mu), \quad y \in \mathscr{Y}, \quad \sum_{y \in \mathscr{Y}} M_{y}(\omega)=I \quad(\bmod \mu),
$$


где $I$ - единичный оператор в $\mathscr{H}$. Распределение вероятностей наблюдаемой $M$ в состоянии $S$ дается формулой

$$
p_{M}(y \mid S)=\int_{\Omega} \operatorname{Tr} S(\omega) M_{y}(\omega) \mu(d \omega) .
$$

3. Классическая пропускная способность каналов с гибридным выходом. Пусть $f(x)$ - неотрицательная функция на множестве $\mathscr{X}=\{x\}$, которое будем называть алфавитом. Обозначим $\mathscr{P}_{1}$ класс конечных распределений вероятностей $\pi$ на $\mathscr{X}$, удовлетворяющих условию

$$
\sum_{x} f(x) \pi_{x} \leqslant E
$$

где $E$ - положительное число. Рассмотрим множество $\mathscr{X}^{n}$ последовательностей букв $\alpha=\left(x_{1}, \ldots, x_{n}\right)$ из $\mathscr{X}$ длины $n$, которые будем называть словами. Введем аддитивное ограничение на входные слова:

$$
f\left(x_{1}\right)+\cdots+f\left(x_{n}\right) \leqslant n E .
$$

Будем обозначать $\mathscr{P}_{n}$ класс распределений на $\mathscr{X}^{n}$, удовлетворяющих условию

$$
\sum_{x_{1}, \ldots, x_{n}}\left(f\left(x_{1}\right)+\cdots+f\left(x_{n}\right)\right) \pi_{x_{1}, \ldots, x_{n}} \leqslant n E .
$$

Классически-гибридным (с-сq) каналом с входным алфавитом $\mathscr{X}$ назовем отображение $\mathscr{S}: x \mapsto S_{x}, S_{x} \in \mathfrak{S}(\Omega, \mathscr{H})$. Если на выходе такого канала происходит измерение наблюдаемой $M=\left\{M_{y}\right\}$, то условная вероятность получить на выходе $y$, если на входе $x$, дается формулой

$$
p_{M}(y \mid x)=\int_{\Omega} \operatorname{Tr} S_{x}(\omega) M_{y}(\omega) \mu(d \omega) .
$$

Блочный канал $\mathscr{S}^{n}$ определяется как отображение слов в сq-состояния на $\mathscr{L}^{\otimes n}: \alpha=\left(x_{1}, \ldots, x_{n}\right) \mapsto S_{x_{1}}\left(\omega_{1}\right) \otimes \cdots \otimes S_{x_{n}}\left(\omega_{n}\right)$. Введем обозначения $\omega^{(n)}=\left(\omega_{1}, \ldots, \omega_{n}\right) \in \Omega^{\times n}, \mu\left(d \omega^{(n)}\right)=\mu\left(d \omega_{1}\right) \cdots \mu\left(d \omega_{n}\right)$.

О п р е д ел е н и е 6 . Кодом длины $n$ и размера $N$ для с-сqканала $\mathscr{S}^{n}$ с ограничением (9) называется пара $(W, M)$, где $W=\left\{\alpha_{i}, i=\right.$ $1, \ldots, N\}$ - набор кодовых слов длины $n$, удовлетворяющих условию (9), $M=\left\{M_{j}, j=0,1, \ldots, N\right\}$ - наблюдаемая в $\mathscr{L}^{\otimes n}$.

Средняя вероятность ошибки кода задается формулой

$$
\bar{P}_{\mathrm{e}}(W, M)=\frac{1}{N} \sum_{j=1}^{N}\left(1-\int_{\Omega^{\times n}} \operatorname{Tr} S_{\alpha_{j}}\left(\omega^{(n)}\right) M_{j}\left(\omega^{(n)}\right) \mu\left(d \omega^{(n)}\right)\right) .
$$

Обозначим $\bar{p}_{e}(n, N)$ инфимум средней вероятности ошибки по всем кодам длины $n$ и размера $N$. 
О п р ед е л е н и е 7. Классической пропускной способностью $C(\Phi)$ с - сq-канала $\mathscr{S}$ с ограничением (9) на входные слова называется супремум достижимых скоростей, т.е. значений $R$, для которых выполняется условие

$$
\lim _{n \rightarrow \infty} \bar{p}_{\mathrm{e}}\left(n, 2^{n R}\right)=0
$$

Пусть теперь дано дискретное распределение вероятностей $\pi=$ $\left\{\pi_{x}\right\}$, рассмотрим соответствующие состояния $S_{x} \in \mathfrak{S}(\Omega, \mathscr{H})$. Обозначим $\bar{S}=\sum_{x} \pi_{x} S_{x}$ - среднее состояние ансамбля. Предположим, что $H_{\mathrm{cq}}(\bar{S})<\infty$, и введем величину

$$
\chi_{\mathrm{cq}}(\pi)=H_{\mathrm{cq}}(\bar{S})-\sum_{x} \pi_{x} H_{\mathrm{cq}}\left(S_{x}\right) .
$$

Следующая лемма обобщает на гибридные системы известное неравенство для квантовых энтропий.

Лемма 3. Для произвольного ансамбля $\left\{\pi_{x}, S_{x}\right\}$, где $\left\{\pi_{x}\right\}-$ pаспределение вероятностей на $\mathscr{X}, S_{x} \in \mathfrak{S}(\Omega, \mathscr{H})$, a $H_{\mathrm{cq}}\left(\sum_{x} \pi_{x} S_{x}\right)<\infty$, выполняются неравенства

$$
0 \leqslant H_{\mathrm{cq}}\left(\sum_{x} \pi_{x} S_{x}\right)-\sum_{x} H_{\mathrm{cq}}\left(S_{x}\right) \leqslant-\sum_{x} \pi_{x} \ln \pi_{x}
$$

Д ок аз а те ль с т в о. Обозначим $\bar{S}=\sum_{x} \pi_{x} S_{x}$. Первое неравенство следует из неотрицательности относительной энтропии, так как $H_{\mathrm{cq}}(S)-\sum_{x} H_{\mathrm{cq}}\left(S_{x}\right)=\sum_{x} \pi_{x} H_{\mathrm{cq}}\left(S_{x} \| \bar{S}\right)$.

Для доказательства второго неравенства воспользуемся свойством энтропии фон Неймана для операторов плотности $A_{x}$ и среднего $\bar{A}=$ $\sum_{x} \pi_{x} A_{x}$ (cм. [10]):

$$
H_{\mathrm{q}}(\bar{A}) \leqslant \sum_{x} \pi_{x} H_{\mathrm{q}}\left(A_{x}\right)-\sum_{x} \pi_{x} \log \pi_{x} .
$$

Обозначим $p(\omega)=\operatorname{Tr} \bar{S}(\omega), \widehat{S}(\omega)=p(\omega)^{-1} \bar{S}(\omega), p_{x}(\omega)=\operatorname{Tr} S_{x}(\omega)$, $\widehat{S}_{x}(\omega)=p_{x}(\omega)^{-1} S_{x}(\omega)$, а $H_{c}(p), H_{c}\left(p_{x}\right)$ - дифференциальные энтропии распределений вероятностей с плотностями $p(\omega)$ и $p_{x}(\omega)$, соответственно. Заметим, что $\widehat{S}(\omega)=\sum_{x} \pi_{x} p_{x}(\omega) p(\omega)^{-1} \widehat{S}_{x}(\omega)$ и воспользуемся свойством (13). Используя (1), имеем

$$
H_{\mathrm{cq}}(\bar{S}) \leqslant H_{\mathrm{c}}(p)+\sum_{x} \pi_{x} \int_{\Omega} p_{x}(\omega)\left(H_{q}\left(\widehat{S}_{x}(\omega)\right)-\log \left(\pi_{x} p_{x}(\omega) p(\omega)^{-1}\right)\right) \mu(d \omega) .
$$

Раскрывая скобки и учитывая, что $\sum_{x} \pi_{x} p_{x}(\omega)=p(\omega)$, а

$$
H_{\mathrm{c}}\left(p_{x}\right)+\int_{\Omega} p_{x}(\omega) H_{\mathrm{q}}\left(\widehat{S}_{x}(\omega)\right) \mu(d \omega)=H_{\mathrm{cq}}\left(S_{x}\right),
$$

получаем требуемое неравенство. Лемма 3 доказана. 
Теорема 1. Пусть $\mathscr{S}: x \mapsto S_{x}, S_{x} \in \mathfrak{S}(\Omega, \mathscr{H})$, - классическигибридный канал с ограничением (9) на входе, удовлетворяющий условию

$$
\sup _{\pi \in \mathscr{P}_{1}} H_{\mathrm{cq}}\left(\sum_{x} \pi_{x} S_{x}\right)<\infty .
$$

Тогда его классическая пропускная способность конечна и дается выражением

$$
C(\mathscr{S})=\sup _{\pi \in \mathscr{P}_{1}} \chi_{\mathrm{cq}}(\pi) .
$$

Д о к а з а т е л ь с т в о. Обозначим $\bar{\chi}_{\text {сq }}$ величину в правой части формулы (14). Сначала докажем неравенство $C(\mathscr{S}) \leqslant \bar{\chi}_{\text {сq }}$, т.е.

$$
\liminf _{n \rightarrow \infty} \bar{p}_{\mathrm{e}}\left(n, 2^{n R}\right)>0 \quad \text { при } R>\bar{\chi}_{\mathrm{cq}} \text {. }
$$

Рассмотрим блочный с-сq канал $\alpha \mapsto S_{\alpha}$, где $\alpha=x^{(n)}-$ слово длины $n$, и наблюдаемую $M^{(n)}$ (в смысле определения 5 ). Тогда, используя монотонность относительной энтропии, для фиксированного разложения единицы можно получить следующую оценку информации Шеннона через относительную энтропию:

$$
\begin{aligned}
I_{n}\left(\pi_{x}^{(n)}, M^{(n)}\right)= & \sum_{x^{(n)}, y^{(n)}} \pi_{x}^{(n)} p_{M}\left(y^{(n)} \mid x^{(n)}\right) \log \frac{p_{M}\left(y^{(n)} \mid x^{(n)}\right)}{\sum_{x^{\prime(n)}} \pi_{x^{\prime}}^{(n)} p_{M}\left(y^{(n)} \mid x^{\prime(n)}\right)} \\
= & \sum_{x^{(n)}, y^{(n)}} \pi_{x}^{(n)} \int_{\Omega^{\times n}} \operatorname{Tr} M_{y^{(n)}\left(\omega^{(n)}\right) S_{x^{(n)}}\left(\omega^{(n)}\right) \mu\left(d \omega^{(n)}\right)} \\
& \times \log \frac{\int_{\Omega^{\times n}} \operatorname{Tr} M_{y^{(n)}}\left(\omega^{(n)}\right) S_{x^{(n)}}\left(\omega^{(n)}\right) \mu\left(d \omega^{(n)}\right)}{\int_{\Omega^{\times n}} \operatorname{Tr} M_{y^{(n)}}\left(\omega^{(n)}\right) \bar{S}_{\pi}^{\otimes n}\left(\omega^{(n)}\right) \mu\left(d \omega^{(n)}\right)} \\
= & \sum_{x} \pi_{x} H_{\mathrm{q}}\left(\Psi^{\otimes n}\left(S_{x}^{\otimes n}\right) \| \Psi^{\otimes n}\left(\bar{S}_{\pi}^{\otimes n}\right)\right) \leqslant \sum_{x} \pi_{x} H_{\mathrm{q}}\left(S_{x}^{\otimes n} \| \bar{S}_{\pi}^{\otimes n}\right) .
\end{aligned}
$$

Здесь $\Psi: \mathfrak{S}(\Omega, \mathscr{H}) \rightarrow \mathfrak{S}(\Omega)$ - канал с квантовым выходом, задаваемый соотношениями

$$
\Psi\left(S_{x}\right)=\sum_{y} p_{M}(y \mid x)\left|e_{y}\right\rangle\left\langle e_{y}\left|, \quad \Psi\left(\bar{S}_{\pi}\right)=\sum_{y}\left(\sum_{x} \pi_{x} p_{M}(y \mid x)\right)\right| e_{y}\right\rangle\left\langle e_{y}\right| .
$$

Заметим, что величина $\bar{\chi}_{\mathrm{cq}}^{(n)}=\sup _{\pi^{(n)} \in \mathscr{P}_{n}} \chi_{\mathrm{cq}}\left(\pi^{(n)}\right)$ аддитивна. Действительно, неравенство $\bar{\chi}_{\mathrm{cq}}^{(n)} \geqslant n \bar{\chi}_{\mathrm{cq}}$ очевидно. Для доказательства обратного неравенства заметим, что для любого распределения $\pi^{(n)} \in$ $\mathscr{P}_{n}$ и маргинальных распределений $\pi^{[i]}$

$$
\chi_{\mathrm{cq}}\left(\pi^{(n)}\right) \leqslant \sum_{i=1}^{n} \chi_{\mathrm{cq}}\left(\pi^{[i]}\right)
$$


в силу субаддитивности полудифференциальной энтропии по отношению к тензорным произведениям (лемма 1 ). Далее $\sum_{i=1}^{n} \chi_{\mathrm{cq}}\left(\pi^{[i]}\right) \leqslant$ $n \chi_{\text {сq }}(\bar{\pi})$ для $\bar{\pi}=n^{-1} \sum_{i=1}^{n} \pi^{[i]}$ в силу того, что $\chi_{\text {сq }}(\pi)$ - вогнутая функция $\pi$. Если $\pi^{(n)} \in \mathscr{P}_{n}$, то $\bar{\pi} \in \mathscr{P}_{1}$, так как неравенство (10) равносильно неравенству

$$
\sum_{x} \sum_{i=1}^{n} \frac{1}{n} f(x) \pi_{x}^{[i]} \leqslant E
$$

Взяв супремум по всем распределениям $\pi^{(n)} \in \mathscr{P}_{n}$, получаем, что $\bar{\chi}_{\text {сq }}^{(n)} \leqslant$ $n \bar{\chi}_{\mathrm{cq}}$.

Таким образом,

$$
\sup _{\pi_{x}^{(n)}, M^{(n)}} I_{n}\left(\pi_{x}^{(n)}, M^{(n)}\right) \leqslant \bar{\chi}_{\mathrm{cq}}^{(n)}=n \bar{\chi}_{\mathrm{cq}} .
$$

Рассмотрим случайную величину, описывающую выходные значения канала при использовании наблюдаемой $M$, при этом слова $\alpha$ кода $(W, M)$ выбираются с равными вероятностями $1 / N$. Слова удовлетворяют ограничению (9), а значит, распределение вероятностей удовлетворяет условию (8). Пусть $N=2^{n R}$, тогда из неравенства Фано [11] и (16) следует, что

$$
n R\left(1-\bar{p}_{\mathrm{e}}\left(n, 2^{n R}\right)\right) \leqslant \sup _{\pi \in \mathscr{P}_{1}} \sup _{M^{(n)}} I_{n}\left(\pi^{(n)}, M^{(n)}\right)+1 \leqslant n \bar{\chi}_{\mathrm{cq}}+1 .
$$

Поэтому, если $R>\bar{\chi}_{\text {сq }}$, то

$$
\bar{p}_{\mathrm{e}}\left(n, 2^{n R}\right) \geqslant 1-\frac{\bar{\chi}_{\mathrm{cq}}}{R}-\frac{1}{n R},
$$

откуда при $n \rightarrow \infty$ следует (15), что доказывает обратное утверждение.

Для доказательства прямого утверждения теоремы кодирования нам понадобятся обобщения понятий типичного и условно типичного проекторов.

Рассмотрим состояние $S \in \mathfrak{S}(\Omega, \mathscr{H})$ и его тензорную степень $S^{\otimes n}$, т.е. функцию $S\left(\omega_{1}\right) \otimes \cdots \otimes S\left(\omega_{n}\right)$ для всех $\omega^{(n)}=\left(\omega_{1}, \ldots, \omega_{n}\right) \in$ $\Omega^{\times n}$. Для каждого $\omega$ рассмотрим спектральное представление оператора $S(\omega): S(\omega)=\sum_{i} \lambda_{i}(\omega)\left|e_{i}(\omega)\right\rangle\left\langle e_{i}(\omega)\right|$. Тогда спектральное представление $S^{\otimes n}\left(\omega^{(n)}\right)$ имеет вид

$$
S^{\otimes n}\left(\omega^{(n)}\right)=\sum_{\lambda_{J}(\omega(n))} \lambda_{J}\left(\omega^{(n)}\right)\left|e_{J}\left(\omega^{(n)}\right)\right\rangle\left\langle e_{J}\left(\omega^{(n)}\right)\right|,
$$

где $\lambda_{J}\left(\omega^{(n)}\right)=\lambda_{j_{1}}\left(\omega_{1}\right) \cdots \lambda_{j_{n}}\left(\omega_{n}\right),\left|e_{J}\left(\omega^{(n)}\right)\right\rangle=\left|e_{j_{1}}\left(\omega_{1}\right)\right\rangle \otimes \cdots \otimes\left|e_{j_{n}}\left(\omega_{n}\right)\right\rangle$.

Пусть $P^{n, \delta}\left(\omega^{(n)}\right)=P^{n, \delta}\left(\omega_{1}, \ldots, \omega_{n}\right)$ - проектор на собственные векторы оператора $S^{\otimes n}\left(\omega^{(n)}\right)$, отвечающий собственным значениям из множества $\left(2^{-n\left(H_{\mathrm{cq}}(S)+\delta\right)} ; 2^{-n\left(H_{\mathrm{cq}}(S)-\delta\right)}\right)$. Такой проектор назовем типичнылм 
проектором для оператора $S^{\otimes n}\left(\omega^{(n)}\right)$. Имеют место следующие свойства типичных проекторов.

1. Размерность $\operatorname{dim} \mathscr{H}^{n, \delta}\left(\omega^{(n)}\right)=\operatorname{Tr} P^{n, \delta}\left(\omega^{(n)}\right)$ удовлетворяет неравенству

$$
\operatorname{dim} \mathscr{H}^{n, \delta}\left(\omega^{(n)}\right) \leqslant 2^{n\left(H_{\mathrm{cq}}(S)+\delta\right)} .
$$

2. Для достаточно больших $n$ выполняется неравенство

$$
\int_{\Omega^{\times n}} \operatorname{Tr} S^{\otimes n}\left(\omega^{(n)}\right)\left(I-P^{n, \delta}\left(\omega^{(n)}\right)\right) \mu\left(d \omega^{(n)}\right)<\varepsilon,
$$

т.е. вклад не $\delta$-типичных векторов может быть сколь угодно малым. Чтобы показать это, рассмотрим вероятностное пространство $\Omega \times \mathbf{N}$ и заданную на нем случайную величину $-\log \lambda_{j}(\omega)$. Соответствующей вероятностной мерой будет

$$
P(A \times\{J\})=\int_{A} \sum_{j \in J} \lambda_{j}(\omega) \mu(d \omega) .
$$

Очевидно, что математическое ожидание $-\log \lambda_{j}(\omega)$ равно

$$
H_{\mathrm{cq}}(S)=-\int_{\Omega} \sum_{j \in J} \lambda_{j}(\omega) \log \lambda_{j}(\omega) \mu(d \omega) .
$$

Обозначим $T^{n, \delta}=\left\{J: 2^{-n\left(H_{\mathrm{cq}}(S)+\delta\right)}<\lambda_{J}\left(\omega^{(n)}\right)<2^{-n\left(H_{\mathrm{cq}}(S)-\delta\right)}\right\}$, тогда

$$
\begin{gathered}
\int_{\Omega^{\times n}} \operatorname{Tr} S^{\otimes n}\left(\omega^{(n)}\right)\left(I-P^{n, \delta}\left(\omega^{(n)}\right)\right) \mu\left(d \omega^{(n)}\right)=P\left\{J \in \bar{T}^{n, \delta}\right\} \\
=P\left\{\left|-\frac{1}{n} \sum_{k=1}^{n} \log \left(\lambda_{j_{k}}\left(\omega_{k}\right)\right)-H_{c q}(S)\right| \geqslant \delta\right\} .
\end{gathered}
$$

Последняя вероятность стремится к нулю по закону больших чисел (для последовательности независимых одинаково распределенных случайных величин $-\log \lambda_{j}(\omega)$, математическое ожидание которых равно $\left.H_{\mathrm{cq}}(S)\right)$.

Введем понятие условно типичного проектора. Рассмотрим слово входного алфавита $\alpha=\left(x_{1}, \ldots, x_{n}\right)$. Тогда блочное состояние $S_{\alpha}=$ $S_{x_{1}} \otimes \cdots \otimes S_{x_{n}}$ задается семейством операторов $S_{x_{1}}\left(\omega_{1}\right) \otimes \cdots \otimes S_{x_{n}}\left(\omega_{n}\right)$. Обозначим

$$
\bar{H}_{\pi}\left(S_{(\cdot)}\right)=\sum_{i} \pi_{x_{i}} H_{\mathrm{cq}}\left(S_{x_{i}}\right) .
$$

Для всех $\omega^{(n)}=\left(\omega_{1}, \ldots, \omega_{n}\right)$ и для каждого кодового слова $\alpha$ можно определить спектральный проектор $P_{\alpha}\left(\omega^{(n)}\right)$ оператора $S_{\alpha}\left(\omega^{(n)}\right)$, отвечающий собственным значениям в интервале $\left(2^{-n\left[\bar{H}_{\pi}\left(S_{(\cdot)}\right)+\delta\right]} ; 2^{-n\left[\bar{H}_{\pi}\left(S_{(\cdot)}\right)-\delta\right]}\right)$, который назовем условно типичным. Имеют место следующие свойства.

1. Для любого слова $\alpha$ и всех $\omega^{(n)}$ выполняется неравенство

$$
P_{\alpha}\left(\omega^{(n)}\right) \leqslant S_{\alpha}\left(\omega^{(n)}\right) \cdot 2^{n\left[\bar{H}_{\pi}\left(S_{(\cdot)}\right)+\delta\right]} .
$$


Это следует непосредственно из определения условно типичного проектора.

2. Пусть $\alpha=\left(x_{1}, \ldots, x_{n}\right)$ выбирается случайно с распределением вероятностей $\pi_{\alpha}=\pi_{x_{1}} \cdots \pi_{x_{n}}$. Обозначим $\mathbf{E}$ математическое ожидание относительно этого распределения. Тогда для любого $\varepsilon>0$ и достаточно больших $n$ имеет место неравенство

$$
\int_{\Omega^{\times n}} \mathbf{E} \operatorname{Tr} S_{\alpha}\left(\omega^{(n)}\right)\left(I-P_{\alpha}\left(\omega^{(n)}\right)\right) \mu\left(d \omega^{(n)}\right) \leqslant \varepsilon .
$$

Действительно, обозначим $T_{\alpha}^{n, \delta}=\left\{J: 2^{-n\left(\bar{H}_{\pi}\left(S_{(\cdot)}\right)+\delta\right)}<\lambda_{J}\left(\omega^{(n)}\right)<\right.$ $\left.2^{-n\left(\bar{H}_{\pi}\left(S_{(\cdot)}\right)-\delta\right)}\right\}$. Тогда левая часть $(17)$ равна

$$
\begin{aligned}
\int_{\Omega^{\times n}} \sum_{\alpha} \pi_{\alpha} \sum_{J \in \bar{T}_{\alpha}^{n, \delta}} \lambda_{J} \mu\left(d \omega^{(n)}\right) & =P\left\{\left|-\frac{1}{n} \log \lambda_{J}-\bar{H}_{\pi}\left(S_{(\cdot)}\right)\right| \geqslant \delta\right\} \\
& =P\left\{\left|-\frac{1}{n} \sum_{k=1}^{n} \log \lambda_{j_{k}}\left(\omega_{k}\right)-\bar{H}_{\pi}\left(S_{(\cdot)}\right)\right| \geqslant \delta\right\} .
\end{aligned}
$$

Последняя вероятность также стремится к нулю по закону больших чисел (для последовательности независимых случайных величин $\left.-\log \lambda_{j}(\omega)\right)$.

Перейдем к доказательству неравенства $C(\mathscr{S}) \geqslant \bar{\chi}_{\text {сq }}$, т.е. покажем, что минимальная средняя вероятность ошибки $\bar{p}_{\mathrm{e}}\left(n, 2^{n R}\right)$ стремится к нулю при $n \rightarrow \infty$, если $R<\bar{\chi}_{\text {сq }}$.

Рассмотрим среднее состояние $\bar{S}_{\pi}$ и для всех $\omega^{(n)}$ типичный проектор $P\left(\omega^{(n)}\right)=P^{n, \delta}\left(\omega^{(n)}\right)$ оператора $\bar{S}_{\pi}^{\otimes n}\left(\omega^{(n)}\right)$. Для данного набора кодовых слов $\left\{\alpha_{1}, \ldots, \alpha_{n}\right\}$ возможно построить условно типичные проекторы $P_{\alpha_{j}}\left(\omega^{(n)}\right)=P_{\alpha_{j}}^{n, \delta}\left(\omega^{(n)}\right)$. По аналогии с доказательством в $[2$, гл. 5] введем субоптимальную наблюдаемую $\left\{M_{j}\left(\omega^{(n)}\right)\right\}$ следующим образом:

$$
\begin{aligned}
M_{j}\left(\omega^{(n)}\right)= & \left(\sum_{i=1}^{N} P\left(\omega^{(n)}\right) P_{\alpha_{i}}\left(\omega^{(n)}\right) P\left(\omega^{(n)}\right)\right)^{-1 / 2} P\left(\omega^{(n)}\right) P_{\alpha_{j}}\left(\omega^{(n)}\right) P\left(\omega^{(n)}\right) \\
& \times\left(\sum_{i=1}^{N} P\left(\omega^{(n)}\right) P_{\alpha_{i}}\left(\omega^{(n)}\right) P\left(\omega^{(n)}\right)\right)^{-1 / 2}, \quad j=1, \ldots, N .
\end{aligned}
$$

Отметим, что для всех $\omega^{(n)}$ набор операторов $\left\{M_{j}\left(\omega^{(n)}\right)\right\}$ является разложением единицы, т.е. определенное таким образом семейство операторов $M$ действительно является наблюдаемой в смысле определения 5 .

Далее для упрощения записи будем использовать для кодовых слов индекс $\alpha$, опуская $j$. Действуя аналогично [2], получаем следующую оценку сверху для средней вероятности ошибки:

$$
\bar{P}_{e}(W, M)
$$




$$
\begin{aligned}
\leqslant \frac{1}{N} \sum_{\alpha=1}^{N} \int_{\Omega^{\times n}}( & 4 \operatorname{Tr} S_{\alpha}\left(\omega^{(n)}\right)\left(I-P\left(\omega^{(n)}\right)\right)+4 \operatorname{Tr} S_{\alpha}\left(\omega^{(n)}\right)\left(I-P_{\alpha}\left(\omega^{(n)}\right)\right) \\
& \left.+\sum_{\alpha^{\prime}: \alpha^{\prime} \neq \alpha} \operatorname{Tr} P\left(\omega^{(n)}\right) S_{\alpha}\left(\omega^{(n)}\right) P\left(\omega^{(n)}\right) P_{\alpha^{\prime}}\left(\omega^{(n)}\right) \mu\left(d \omega^{(n)}\right)\right) .
\end{aligned}
$$

Теперь пусть $\pi=\left\{\pi_{x}\right\}$ - распределение вероятностей на $\mathscr{X}$, удовлетворяющее условию (8). Применим процедуру случайного кодирования, предполагая, что слова $\alpha=\left(x_{1}, \ldots, x_{n}\right)$ выбираются случайным образом, независимо друг от друга, с распределением вероятностей $\pi_{\alpha}=\pi_{x_{1}} \cdots \pi_{x_{n}}$, и заметим, что это распределение будет принадлежать классу $\mathscr{P}_{n}$.

Тогда для всех $\omega^{(n)}$ имеем

$$
\mathbf{E} S_{\alpha}\left(\omega^{(n)}\right)=\sum_{x_{1}, \ldots, x_{n}} \pi_{x_{1}} \cdots \pi_{x_{n}} S_{x_{1}}\left(\omega_{1}\right) \otimes \cdots \otimes S_{x_{n}}\left(\omega_{n}\right)=\bar{S}_{\pi}^{\otimes n}\left(\omega^{(n)}\right)
$$

(здесь математическое ожидание берется относительно распределения вероятностей $\left.\pi_{\alpha}\right)$. Усреднив предыдущее неравенство, получаем:

$$
\begin{aligned}
\mathbf{E} \bar{P}_{\mathrm{e}}(W, M) \leqslant & 4 \int_{\Omega^{\times n}} \operatorname{Tr} \bar{S}_{\pi}^{\otimes n}\left(\omega^{(n)}\right)\left(I-P\left(\omega^{(n)}\right)\right) \mu\left(d \omega^{(n)}\right) \\
& +4 \int_{\Omega^{\times n}} \mathbf{E} \operatorname{Tr} S_{\alpha}\left(\omega^{(n)}\right)\left(I-P_{\alpha}\left(\omega^{(n)}\right)\right) \mu\left(d \omega^{(n)}\right) \\
& +(N-1) \int_{\Omega^{\times n}} \operatorname{Tr} \bar{S}_{\pi}^{\otimes n}\left(\omega^{(n)}\right) P\left(\omega^{(n)}\right) \mathbf{E} P_{\alpha^{\prime}}\left(\omega^{(n)}\right) \mu\left(d \omega^{(n)}\right) .
\end{aligned}
$$

По свойствам типичных и условно типичных проекторов первые два слагаемых меньше $\varepsilon$ для достаточно больших $n$. Для оценки третьего слагаемого заметим, что

$$
\operatorname{Tr} \bar{S}_{\pi}^{\otimes n}\left(\omega^{(n)}\right) P\left(\omega^{(n)}\right) \mathbf{E} P_{\alpha^{\prime}}\left(\omega^{(n)}\right) \leqslant\left\|\bar{S}_{\pi}^{\otimes n}\left(\omega^{(n)}\right) P\left(\omega^{(n)}\right)\right\| \operatorname{Tr} \mathbf{E} P_{\alpha^{\prime}}\left(\omega^{(n)}\right) .
$$

Очевидно, что справедлива оценка

$$
\left\|\bar{S}_{\pi}^{\otimes n}\left(\omega^{(n)}\right) P\left(\omega^{(n)}\right)\right\| \leqslant 2^{-n\left[H_{c q}\left(\bar{S}_{\pi}\right)-\delta\right]}
$$

так как собственные значения оператора $\bar{S}_{\pi}^{\otimes n}\left(\omega^{(n)}\right) P\left(\omega^{(n)}\right)$ ограничены сверху величиной, стоящей в правой части.

С другой стороны,

$\operatorname{Tr} \mathbf{E} P_{\alpha^{\prime}}\left(\omega^{(n)}\right)=\mathbf{E} \operatorname{Tr} P_{\alpha^{\prime}}\left(\omega^{(n)}\right) \leqslant \operatorname{Tr} S_{\alpha^{\prime}}\left(\omega^{(n)}\right) \cdot 2^{n\left[\bar{H}_{\pi}\left(S_{(\cdot)}\right)+\delta\right]} \leqslant 2^{n\left[\bar{H}_{\pi}\left(S_{(\cdot)}\right)+\delta\right]}$.

Интегрируя по $\Omega^{\times n}$ и учитывая, что $H_{\mathrm{cq}}\left(\bar{S}_{\pi}\right)-\bar{H}_{\pi}\left(S_{(\cdot)}\right)=\chi_{\mathrm{cq}}\left(\pi_{\alpha}\right)$, имеем

$$
\bar{p}_{\mathrm{e}}(n, N) \leqslant \mathbf{E} \bar{P}_{\mathrm{e}}(W, M) \leqslant 8 \varepsilon+N \cdot 2^{-n\left[\chi_{\mathrm{cq}}\left(\pi_{\alpha}\right)-2 \delta\right]} .
$$


Теперь модифицируем вероятностную меру таким образом, чтобы ненулевые вероятности приписывались только словам, удовлетворяющим условию $(9)$. Обозначим $\nu_{n}=P\left(\sum_{k=1}^{n} f\left(i_{k}\right) \leqslant n E\right)$ и введем меру $\widetilde{P}$ следующим образом:

$$
\widetilde{P}\left(\alpha=\left(x_{1}, \ldots, x_{n}\right)\right)= \begin{cases}\nu_{n}^{-1} \pi_{x_{1}} \cdots \pi_{x_{n}}, & \text { если } \sum_{i=1}^{n} f\left(x_{i}\right) \leqslant n E, \\ 0 & \text { в противном случае. }\end{cases}
$$

Рассуждая аналогично [2], заметим, что если взять математическое ожидание $\bar{P}_{\mathrm{e}}(W, M)$ относительно меры $\widetilde{P}$ (обозначим его $\widetilde{\mathbf{E}}$ ), то

$$
\widetilde{\mathbf{E}} \inf _{M} \bar{P}_{\mathrm{e}}(W, M) \leqslant 4 \mathbf{E} \inf _{M} \bar{P}_{\mathrm{e}}(W, M) .
$$

Теперь выберем распределение $\pi_{\alpha}=\pi_{\alpha}^{0}$ таким образом, чтобы выполнялось неравенство $\chi_{\text {сq }}\left(\pi_{\alpha}^{0}\right) \geqslant \bar{\chi}_{c q}-\delta$. Тогда, если $R \leqslant \bar{\chi}_{\text {сq }}-4 \delta$, а $N=2^{n R}$, то

$$
\mathbf{E} \bar{P}_{\mathrm{e}}(W, M) \leqslant 8 \epsilon+2^{-n \delta} \rightarrow 0 \quad \text { при } n \rightarrow \infty .
$$

Следовательно, $\widetilde{\mathbf{E}} \inf _{M} \bar{P}_{\mathrm{e}}(W, M)$ также стремится к нулю при $R<$ $\bar{\chi}_{\text {сq }}-4 \delta$. Мера $\widetilde{P}$ сконцентрирована на словах, удовлетворяющих условию (9), поэтому найдется последовательность кодов размера $N=2^{n R}$, для которых $\bar{P}_{\mathrm{e}}(W, M)$ стремится к нулю при $n \rightarrow \infty$. Тем самым установлено неравенство $C(\mathscr{S}) \geqslant \bar{\chi}_{c q}$. Теорема 1 доказана.

П р и м е ч а н и е. Доказательство теоремы 1 можно обобщить на случай, когда выполняется условие $H_{\text {сq }}\left(S_{x}\right)<\infty$, без предположения о конечности $H_{\text {сq }}(\bar{S})$. В этом случае классическая пропускная способность может принимать как конечные, так и бесконечные значения.

Теперь пусть $\mathscr{H}_{A}, \mathscr{H}_{B}$ - гильбертовы пространства. Назовем $\kappa a-$ налом с квантовым входом и классически-квантовым выходом (q-сqканалом) линейное, вполне положительное отображение из $\mathfrak{S}\left(\mathscr{H}_{A}\right)$ в $\mathfrak{S}\left(\Omega, \mathscr{H}_{B}\right)$.

Рассмотрим q-сq-канал $\Phi: \mathfrak{S}\left(\mathscr{H}_{A}\right) \rightarrow \mathfrak{S}\left(\Omega, \mathscr{H}_{B}\right)$. Дадим определение классической пропускной способности при аддитивном ограничении на входе.

Пусть $F-$ самосопряженный положительный, вообще говоря неограниченный, оператор в пространстве $\mathscr{H}_{A}$, не пропорциональный единичному, со спектральным разложением $F=\int_{0}^{\infty} x d E(x)$, где $E(x)-$ спектральная функция оператора $F$. Рассмотрим множество состояний $S \in \mathfrak{S}\left(\mathscr{H}_{A}\right)$, удовлетворяющих условию

$$
\operatorname{Tr} S F \leqslant E,
$$

где $E$ - некоторая положительная постоянная, причем след в левой части (18) понимается в смысле $\operatorname{Tr} S F=\int_{0}^{\infty} x d \operatorname{Tr} S E(x)$ (более подробно cм. [5]). 
Пусть $\mathscr{A}$ обозначает множество состояний $S$, удовлетворяющих условию (18). Мы и будем рассматривать канал $\Phi$ с этим ограничением на входные состояния. В дальнейшем нам понадобится тот факт (см. [5]), что если оператор $F$ удовлетворяет условию

$$
\operatorname{Tr} \exp (-\beta F)<\infty \quad \text { для всех } \beta>0 \text {, }
$$

то $H(S)<\infty$ для $S \in \mathscr{A}$.

Для канала $\Phi^{\otimes n}$ соответствующий оператор есть

$$
F^{(n)}=F \otimes I \otimes \cdots \otimes I+\cdots+I \otimes I \otimes \cdots \otimes F
$$

через $\mathscr{A}^{(n)}$ обозначим множество состояний $S^{(n)}$ из $\mathfrak{S}\left(\mathscr{H}^{\otimes n}\right)$, удовлетворяющих условию

$$
\operatorname{Tr} S^{(n)} F^{(n)} \leqslant n E .
$$

О п р е д е л е н и е 8 . Кодом длины $n$ и размера $N$ для q-сqканала с ограничением называется пара:

1) семейство $\Sigma^{(n)}=\left\{S_{\alpha}^{(n)}\right\}$ состояний из $\mathfrak{S}\left(\mathscr{H}_{A}\right)$, удовлетворяющих ограничению (20);

2) разложение единицы $M=\left\{M_{j}, j=0,1, \ldots, N\right\}$ в $\mathscr{L}^{\otimes n}$ (в смысле определения 5).

Вероятность ошибки такого кода равна

$$
\lambda_{\mathrm{e}}\left(\Sigma^{(n)}, M^{(n)}\right)=\max _{\alpha=1, \ldots, N}\left(1-\int_{\Omega^{\times n}} \operatorname{Tr}\left[\Phi^{\otimes n}\left(S_{\alpha}^{(n)}\left(\omega^{(n)}\right)\right)\right] M_{\alpha}\left(\omega^{(n)}\right) \mu\left(d \omega^{(n)}\right)\right) .
$$

Минимальную вероятность ошибки по всевозможным кодам длины $n$ и размера $N$ обозначим $\bar{\lambda}_{\mathrm{e}}(n, N)$. Классическая пропускная способность $\bar{C}_{\text {сq }}(\Phi)$ такого канала есть точная верхняя грань скоростей передачи $R$, для которых $\lim _{n \rightarrow \infty} \bar{\lambda}_{\mathrm{e}}\left(n, 2^{n R}\right)=0$.

Обозначим $\mathfrak{P}_{n}$ множество пар $\left(\pi^{(n)}, \Sigma^{(n)}\right)$, где $\pi_{\alpha}^{(n)}$ - вероятности состояний $S_{\alpha}^{(n)}$, удовлетворяющих условию

$$
\sum_{\alpha=1}^{N} \pi_{\alpha}^{(n)} \operatorname{Tr} S_{\alpha}^{(n)} F^{(n)} \leqslant n E .
$$

Пусть $\left\{\pi_{\alpha}^{(n)}\right\}$ - распределение вероятностей на входных состояниях $\left\{S_{\alpha}^{(n)}\right\}$ и $\pi(i \mid \alpha)=\int_{\Omega^{\times n}} \operatorname{Tr} \Phi^{\otimes n}\left(S_{\alpha}^{(n)}\left(\omega^{(n)}\right)\right) M_{i}\left(\omega^{(n)}\right) \mu\left(d \omega^{(n)}\right)$ - переходные вероятности. Тогда можно рассмотреть шенноновскую информацию $I_{n}\left(\pi^{(n)}, \Sigma^{(n)}, M^{(n)}\right)$, найдя совместное распределение входа и выхода.

Определим величину

$$
\bar{C}_{\mathrm{cq}}^{(n)}(\Phi)=\sup _{\left(\pi^{(n)}, \Sigma^{(n)}\right) \in \mathfrak{P}_{n}} \chi_{\mathrm{cq}}\left(\left\{\pi_{\alpha}^{(n)}\right\},\left\{\Phi^{\otimes n}\left(S_{\alpha}^{(n)}\left(\omega^{n}\right)\right)\right\}\right) .
$$


Если $\Sigma^{(n)} \in \mathscr{A}^{(n)}$, то $\left(\pi^{(n)}, \Sigma^{(n)}\right) \in \mathfrak{P}^{(n)}$, и в силу квантовой энтропийной границы [2]

$$
I_{n}\left(\pi^{(n)}, \Sigma^{(n)}, M^{(n)}\right) \leqslant \bar{C}_{\mathrm{cq}}^{(n)}(\Phi) .
$$

Имеет место следующая теорема кодирования, доказательство которой аналогично доказательству предложения 3 в [5] с применением теоремы 1 к с-сq каналу $\widetilde{\Phi}: \alpha \mapsto \Phi^{\otimes n}\left(S_{\alpha}\right)$.

Теорема 2. Пусть $\mathscr{H}_{A}, \mathscr{H}_{B}-$ гильбертовь пространства и канал $\Phi$ действует из $\mathfrak{S}\left(\mathscr{H}_{A}\right)$ в $\mathfrak{S}\left(\Omega, \mathscr{H}_{B}\right)$. Пусть также входные состояния $S^{(n)}$ канала $\Phi^{(n)}$ удовлетворяют ограничению

$$
\operatorname{Tr} S^{(n)} F^{(n)} \leqslant n E,
$$

а канал $\Phi$ удовлетворяет условию

$$
\sup _{S: \operatorname{Tr} S F \leqslant E} H_{\mathrm{cq}}(\Phi(S))<\infty .
$$

Классическая пропускная способность такого канала конечна и равна

$$
\bar{C}(\Phi)=\lim _{n \rightarrow \infty} \frac{1}{n} \bar{C}_{\mathrm{cq}}^{(n)}\left\{\Phi^{\otimes n}\right\} .
$$

4. Теорема о классической пропускной способности измерительного канала с использованием сцепленности. Пусть $\mathscr{H}_{A}$ - гильбертово пространство и $\Phi$ - квантовый канал, действующий из $\mathfrak{S}\left(\mathscr{H}_{A}\right)$ в $\mathfrak{S}\left(\mathscr{H}_{A^{\prime}}\right)$. Дадим определение пропускной способности с использованием сцепленности между входом и выходом канала $\Phi$ при аддитивном ограничении на входе.

Опишем протокол передачи информации с использованием сцепленного состояния. Предполагается, что передатчик $A$ и приемник $B$ находятся в сцепленном состоянии, описываемом вектором $|\psi\rangle=\sum_{j} \lambda_{j}\left|e_{j}\right\rangle \otimes$ $\left|e_{j}\right\rangle$, где $\left\{\left|e_{i}\right\rangle \otimes\left|e_{j}\right\rangle\right\}$ - ортонормированный базис в пространстве $\mathscr{H}_{A} \otimes \mathscr{H}_{B}$ и сцепленность означает, что все $\lambda_{j}$ строго больше 0 , т.е. вектор $|\psi\rangle$ не является произведением векторов в пространствах $\mathscr{H}_{A}, \mathscr{H}_{B}$. Предположим, что энтропия частичных следов состояния $S_{A B}=|\psi\rangle\langle\psi|$ конечна:

$$
H_{\mathrm{q}}\left(S_{A}\right)=H_{\mathrm{q}}\left(S_{B}\right)<\infty .
$$

Пусть $\mathscr{X}$ - конечный алфавит. Участник $A$ кодирует классический сигнал $x \in \mathscr{X}$, появляющийся с вероятностью $\pi_{x}$, в кодирующий канал $\mathscr{E}_{x}$, действующий в $\mathscr{H}_{A}$. Далее $\mathscr{E}_{x}$ применяется к соответствующей части состояния $S_{A B}$, после чего совместное состояние $A$ и $B$ можно описать как

$$
\left(\mathscr{E}_{x} \otimes \operatorname{Id}_{B}\right)|\psi\rangle\left\langle\psi\left|=\sum_{j, k} \lambda_{j} \lambda_{k} \mathscr{E}_{x}\left(\left|e_{j}\right\rangle\left\langle e_{k}\right|\right) \otimes\right| e_{j}\right\rangle\left\langle e_{k}\right|
$$


После передачи по каналу $\Phi$ состояния системы $A^{\prime} B$ описываются операторами плотности

$$
\sigma_{x}=\sum_{j, k} \lambda_{j} \lambda_{k} \Phi\left[\mathscr{E}_{x}\left(\left|e_{j}\right\rangle\left\langle e_{k}\right|\right)\right] \otimes\left|e_{j}\right\rangle\left\langle e_{k}\right| .
$$

Предполагается, что участник $B$ может производить измерения наблюдаемых в системе $A^{\prime} B$, извлекая таким образом информацию о сигнале $x$.

Возможно применение блочного кодирования, тогда кодированные состояния, передаваемые через канал $\Phi^{\otimes n} \otimes \operatorname{Id}_{B}^{\otimes n}$, имеют вид

$$
S_{\alpha}^{(n)}=\left(\mathscr{E}_{\alpha}^{(n)} \otimes \operatorname{Id}_{B}^{\otimes n}\right)\left[S_{A B}^{(n)}\right],
$$

где $S_{A B}^{(n)}$ - чистое сцепленное состояние для $n$ экземпляров системы $A B$, удовлетворяющее условию $H\left(S_{B}^{(n)}\right)<\infty, \alpha-$ классическое сообщение (например, слово в алфавите $\mathscr{X}), \alpha \mapsto \mathscr{E}_{\alpha}^{(n)}$ - кодирования для $n$ экземпляров системы $A$. На входные состояния канала $\Phi^{\otimes n}$ налагается ограничение (20), которое эквивалентно аналогичному ограничению для канала $\Phi^{\otimes n} \otimes \operatorname{Id}_{B}^{\otimes n}$ с операторами $F^{(n)} \otimes I_{B}^{\otimes n}$. Обозначим $\mathfrak{P}_{n}^{A B}$ совокупность пар $\left(\pi^{(n)}, \Sigma^{(n)}\right)$, где $\pi^{(n)}=\left\{\pi_{\alpha}^{(n)}\right\}$ - конечное распределение вероятностей, а $\Sigma^{(n)}=\left\{S_{\alpha}^{(n)}\right\}-$ множество состояний вида (23), удовлетворяющих ограничению

$$
\sum_{\alpha} \pi_{\alpha}^{(n)} \operatorname{Tr} S_{\alpha}^{(n)}\left(F^{(n)} \otimes I_{B}^{\otimes n}\right) \leqslant n E .
$$

Для канала $\Phi$ с ограничением (18) рассмотрим величину

$$
C_{\mathrm{ea}}^{(n)}\left(\Phi^{\otimes n}, \mathscr{A}^{(n)}\right)=\sup _{\left(\pi^{(n)}, \Sigma^{(n)}\right) \in \mathfrak{P}_{n}^{A B}} \chi\left(\left\{\pi_{\alpha}^{(n)}\right\} ;\left\{\left(\Phi^{\otimes n} \otimes \operatorname{Id}_{B}^{\otimes n}\right) S_{\alpha}^{(n)}\right\}\right),
$$

где величина $\chi\left(\left\{\pi_{x}\right\} ;\left\{S_{x}\right\}\right)=H_{\mathrm{q}}\left(\sum_{x} \pi_{x} S_{x}\right)-\sum_{x} \pi_{x} H_{\mathrm{q}}\left(S_{x}\right)-$ квантовый аналог информации Шеннона (см., например, [2, гл. 5]). Классическую пропускную способность с использованием сцепленности канала $\Phi$ с ограничением $\mathscr{A}$ зададим соотношением

$$
C_{\text {ea }}(\Phi, \mathscr{A})=\lim _{n \rightarrow \infty} \frac{1}{n} C_{\mathrm{ea}}^{(n)}\left(\Phi^{\otimes n}, \mathscr{A}^{(n)}\right) .
$$

K такому определению приводит определение в терминах достижимых скоростей передачи (см. [5]).

О п р е д е л е н и е 9 . Взаимной информацией канала $\Phi$ на состоянии $S \in \mathfrak{S}(\mathscr{H})$ называется величина

$$
I(S, \Phi)=H_{\mathrm{q}}(S)+H_{\mathrm{q}}(\Phi(S))-H_{\mathrm{q}}\left(\Phi \otimes \operatorname{Id}_{R}\left(\left|\psi_{S}\right\rangle\left\langle\psi_{S}\right|\right)\right),
$$

где $\left|\psi_{S}\right\rangle\left\langle\psi_{S}\right|$ - очищение состояния $S$. 
Для квантовых каналов, действующих в конечномерных пространствах, имеет место теорема кодирования, дающая аналитическое выражение для $C_{\text {еа }}(\Phi)$ через взаимную информацию [3]:

$$
C_{\text {ea }}(\Phi)=\max _{S_{A}} I\left(S_{A} ; \Phi\right) .
$$

В случае бесконечномерных пространств в работе [5] доказана соответствующая теорема для канала $\Phi$ с ограничением (18), а именно:

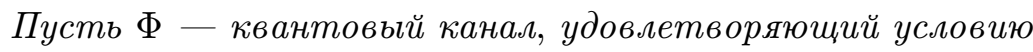

$$
\sup _{S: \operatorname{Tr} S F \leqslant E} H_{\mathrm{q}}(\Phi[S])<\infty
$$

c оператором $F$, удовлетворяющим условию (19). Тогда его пропускная способность с использованием сцепленного состояния при ограничении (18) конечна и равна

$$
C_{\text {ea }}(\Phi, \mathscr{A})=\sup _{S: \operatorname{Tr} S F \leqslant E} I(S, \Phi) .
$$

Этот результат применим к случаю измерительных каналов с не более чем счетным множеством исходов, но не охватывает измерения с непрерывными исходами (такие как отмеченные выше квантовая томография в конечномерном пространстве, оптическое гетеродинирование с ограничением на энергию входного сигнала) [4], представляющие большой интерес. Нашей целью является получение соответствующего результата для измерительных каналов с произвольным множеством исходов.

Введем понятие измерительного канала $\mathscr{M}$ для случая, когда множество исходов - некоторое измеримое пространство $\Omega$. Рассмотрим семейство положительных ограниченных операторов $V(\omega)$, слабо измеримых относительно меры $\mu$ и удовлетворяющих условию

$$
\int_{\Omega} V(\omega)^{*} V(\omega) \mu(d \omega)=I
$$

Зададим эффективное измерение $\mathfrak{M}$ (более подробное определение эффективного измерения см. в [12]), множество исходов которого - измеримое пространство $\Omega$, как отображение, ставящее в соответствие каждому состоянию $S \in \mathfrak{S}(\mathscr{H})$ распределение вероятностей с плотностью

$$
\operatorname{Tr} S M(\omega), \quad \text { где } M(\omega)=V(\omega)^{*} V(\omega)
$$

относительно меры $\mu$, и семейство апостериорных состояний $\widehat{S}(\omega)=$ $(\operatorname{Tr} S M(\omega))^{-1} V(\omega) S V(\omega)^{*}$. 
О п р е д е л е н и е 10. Измерительныл каналом $\mathscr{M}$ называется отображение состояния $S \in \mathfrak{S}(\mathscr{H})$ в распределение вероятностей $(25)$, где $M=\{M(d \omega)\}$ - наблюдаемая с множеством исходов $\Omega$, отвечающая описанному выше измерению.

Протокол передачи информации с использованием сцепленности применим и для измерительного канала $\mathscr{M}$, однако при этом в распоряжении участника $B$ оказывается гибридная система $A^{\prime} B$, где $A^{\prime}=\Omega$ - классическая система на выходе измерительного канала. Таким образом, после измерения $\mathfrak{M}$ состояние в гибридной системе описывается следующим образом:

$$
\sigma_{x}(d \omega)=\sum_{j, k} \lambda_{j} \lambda_{k}\left[\operatorname{Tr} \mathscr{E}_{x}\left(\left|e_{j}\right\rangle\left\langle e_{k}\right|\right) M(d \omega)\right]\left|e_{j}\right\rangle\left\langle e_{k}\right| .
$$

Для канала $\mathscr{M}$ с ограничением (18) на входные состояния рассмотрим величину, аналогичную (24):

$$
\begin{aligned}
\widetilde{C}_{\mathrm{ea}}^{(n)}\left(\mathscr{M}^{\otimes n}, \mathscr{A}^{(n)}\right)= & \sup _{\left(\pi^{(n)}, \Sigma^{(n)}\right) \in \mathfrak{P}_{n}^{A B}} \chi_{\mathrm{cq}}\left(\left\{\pi_{\alpha}^{(n)}\right\} ;\left\{\left(\mathscr{M}^{\otimes n} \otimes \operatorname{Id}_{B}^{\otimes n}\right) S_{\alpha}^{(n)}\right\}\right) \\
= & \sup _{\left(\pi^{(n)}, \Sigma^{(n)}\right) \in \mathfrak{P}_{n}^{A B}}\left[H_{\mathrm{cq}}\left(\sum_{\alpha} \pi_{\alpha}^{(n)}\left(\mathscr{M}^{\otimes n} \otimes \operatorname{Id}_{B}^{\otimes n}\right) S_{\alpha}^{(n)}\right)\right. \\
& \left.-\sum_{\alpha} \pi_{\alpha}^{(n)} H_{c q}\left(\left(\mathscr{M}^{\otimes n} \otimes \operatorname{Id}_{B}^{\otimes n}\right) S_{\alpha}^{(n)}\right)\right] .
\end{aligned}
$$

Из теоремы 2 следует, что классическая пропускная способность с использованием сиепленного состояния для квантово-классического канала $\mathscr{M}$ при ограничении (18) дается соотношением

$$
C_{\text {ea }}(\mathscr{M}, \mathscr{A})=\lim _{n \rightarrow \infty} \frac{1}{n} \widetilde{C}_{\text {ea }}^{(n)}\left(\mathscr{M}^{\otimes n}, \mathscr{A}^{(n)}\right) .
$$

Рассмотрим следующую характеристику измерения $\mathfrak{M}$ :

$$
\operatorname{ER}(S, \mathfrak{M})=H_{\mathrm{q}}(S)-\int_{\Omega}(\operatorname{Tr} S M(\omega)) H_{\mathrm{q}}(\widehat{S}(\omega)) \mu(d \omega)
$$

называемую редукиией энтропии. В [12] показано, что для измерительных каналов с дискретным выходом $I(S, \Phi)$ совпадает с $\operatorname{ER}(S, \mathfrak{M})$. Докажем теорему, дающую выражение для $C_{\text {еа }}(\mathscr{M})$ в случае измерительных каналов с непрерывным пространством исходов через $\operatorname{ER}(S, \mathfrak{M})$.

Теорема 3. Пусть $\mathscr{M}$ - измерительньй канал с ограничением на входе вида $\operatorname{Tr} S_{A} F \leqslant E$, где $S_{A}$ - входное состояние. Предположим, что оператор $F$ удовлетворяет условию (19), а канал $\mathscr{M}$ дополнительно удовлетворяет условию

$$
\sup _{S_{A}: \operatorname{Tr} S_{A} F \leqslant E} H_{\mathrm{c}}\left(\mathscr{M}\left(S_{A}\right)\right)<\infty
$$


где $H_{\mathrm{c}}\left(\mathscr{M}\left(S_{A}\right)\right)$ - классическая дифференииальная энтропия распределения вероятностей (25). Тогда

$$
C_{\text {ea }}(\mathscr{M}, \mathscr{A})=\sup _{S_{A}: \operatorname{Tr} S_{A} F \leqslant E} \operatorname{ER}\left(S_{A}, \mathfrak{M}\right) .
$$

Д о к а з а т е л ь с т в о. Сначала докажем неравенство

$$
C_{\text {ea }}(\mathscr{M}, \mathscr{A}) \leqslant \sup _{S_{A}: \operatorname{Tr} S_{A} F \leqslant E} \operatorname{ER}\left(S_{A}, \mathfrak{M}\right),
$$

для чего покажем, что $\widetilde{C}_{\text {ea }}^{(1)}(\mathscr{M}, \mathscr{A}) \leqslant \sup _{S_{A}: \operatorname{Tr} S_{A} F \leqslant E} \operatorname{ER}\left(S_{A}, \mathfrak{M}\right)$.

Пусть $\mathscr{E}_{A}$ - кодирование, используемое в системе $A$, и пусть $S_{A B}-$ исходное состояние системы $A B$ :

$$
S_{A B}=\sum_{i, j} \lambda_{i} \lambda_{j}\left|e_{i}\right\rangle\left\langle e_{j}|\otimes| e_{i}\right\rangle\left\langle e_{j}\right|
$$

где $\left\{\left|e_{i}\right\rangle \otimes\left|e_{j}\right\rangle\right\}$ - ортонормированный базис в пространстве $\mathscr{H}_{A} \otimes \mathscr{H}_{B}$.

Разделенным между системами $A$ и $B$ состоянием после кодирования будет

$$
S_{A B}^{x}=\left(\mathscr{E}_{A}^{x} \otimes \operatorname{Id}_{B}\right) S_{A B}
$$

с частичными состояниями $S_{A}^{x}=\mathscr{E}_{A}^{x}\left(S_{A}\right)$ и $S_{B}^{x}=S_{B}$ (заметим, что состояние в системе $B$ не изменяется).

Докажем неравенство

$H_{\mathrm{cq}}\left(\sum_{x} \pi_{x}\left(\mathscr{M} \otimes \operatorname{Id}_{B}\right) S_{A B}^{x}\right)-\sum_{x} \pi_{x} H_{\mathrm{cq}}\left(\mathscr{M} \otimes \operatorname{Id}_{B}\left(S_{A B}^{x}\right)\right) \leqslant \operatorname{ER}\left(\sum_{x} \pi_{x} S_{A}^{x}, \mathfrak{M}\right)$.

Заметим, что ограничения на входные состояния канала приводят к следующему ограничению на кодирования:

$$
\operatorname{Tr} \sum_{x} \pi_{x} S_{A}^{x} F \leqslant E .
$$

Для первого слагаемого в левой части (30) имеет место оценка

$$
\begin{aligned}
H_{\mathrm{cq}} & \left(\sum_{x} \pi_{x}\left(\mathscr{M} \otimes \operatorname{Id}_{B}\right) S_{A B}\right) \\
& =\int_{\Omega} H_{\mathrm{q}}\left(\sum_{x} \pi_{x} \sum_{i, j} \lambda_{i} \lambda_{j}\left[\operatorname{Tr} M(\omega) \mathscr{E}_{A}^{x}\left|e_{i}\right\rangle\left\langle e_{j}\right|\right]\left|e_{i}\right\rangle\left\langle e_{j}\right|\right) \mu(d \omega) \\
& \leqslant H_{\mathrm{c}}(p)+H_{\mathrm{q}}\left(S_{B}\right),
\end{aligned}
$$

где $H_{\mathrm{c}}(p)$ - энтропия распределения вероятностей с плотностью $p(\omega)=$ $\operatorname{Tr} V(\omega) \bar{S}_{A} V(\omega)^{*}, \bar{S}_{A}=\sum_{x} \pi_{x} S_{A}^{x}$. Заметим, что $H_{\mathrm{c}}(p)=H_{\mathrm{c}}\left(\mathscr{M}\left(\bar{S}_{A}\right)\right)-$ выходная энтропия канала $\mathscr{M}$ на состоянии $\bar{S}_{A}$ и $H_{c}\left(\mathscr{M}\left(\bar{S}_{A}\right)\right)<\infty$. 
Для остальных слагаемых в (30) имеем

$$
\begin{aligned}
\sum_{x} & \pi_{x}\left[H_{\mathrm{q}}\left(S_{B}\right)-H_{\mathrm{cq}}\left(\mathscr{M} \otimes \operatorname{Id}_{B}\left(S_{A B}^{x}\right)\right)\right] \\
& =\sum_{x} \pi_{x}\left[H_{\mathrm{q}}\left(S_{B}\right)-\int_{\Omega} H_{\mathrm{q}}\left(\sum_{i, j} \lambda_{i} \lambda_{j}\left[\operatorname{Tr} M(\omega) \mathscr{E}_{A}^{x}\left|e_{i}\right\rangle\left\langle e_{j}\right|\right]\left|e_{i}\right\rangle\left\langle e_{j}\right|\right) \mu(d \omega)\right] \\
& =-\sum_{x} \pi_{x} H_{\mathrm{cq}}\left(\Omega^{x} \mid B\right)
\end{aligned}
$$

в смысле определения 4. Здесь мы используем обозначение $\Omega^{x}$, чтобы показать, что с-состояние $\mathscr{M} \otimes \operatorname{Id}_{B}\left(S_{A B}^{x}\right)$ зависит от $x$ (q-состояние равно $S_{B}$ для всех $\left.x\right)$.

Для каждого $x$ рассмотрим очищающее пространство $\mathscr{H}_{R^{x}}$, т.е. такую систему $R^{x}$, что состояние $S_{A R^{x}}$ - чистое. Например, можно положить $R^{x}=B E^{x}$, где $E^{x}$ - эталонная система для $A^{x} B$. Тогда

$$
\begin{gathered}
H_{\mathrm{q}}\left(S_{A}^{x}\right)-H_{\mathrm{cq}}\left(\mathscr{M} \otimes \operatorname{Id}_{R^{x}}\left(S_{A R^{x}}^{x}\right)\right)=H_{\mathrm{q}}\left(A^{x}\right)-H_{\mathrm{cq}}\left(\Omega^{x} R^{x}\right) \\
=H_{\mathrm{q}}\left(B E^{x}\right)-H_{\mathrm{cq}}\left(\Omega^{x} B E^{x}\right)=-H_{\mathrm{cq}}\left(\Omega^{x} \mid B E^{x}\right) .
\end{gathered}
$$

По лемме 2 для любого $x$ выполняется неравенство

$$
-H_{\mathrm{cq}}\left(\Omega^{x} \mid B E^{x}\right) \geqslant-H_{\mathrm{cq}}\left(\Omega^{x} \mid B\right),
$$

т.e.

$$
\begin{aligned}
\sum_{x} \pi_{x}( & \left.H_{\mathrm{q}}\left(S_{B}\right)-H_{\mathrm{cq}}\left(\mathscr{M} \otimes \operatorname{Id}_{B}\left(S_{A B}^{x}\right)\right)\right) \\
& \leqslant \sum_{x} \pi_{x}\left(H_{\mathrm{q}}\left(S_{A}^{x}\right)-H_{\mathrm{cq}}\left(\mathscr{M} \otimes \operatorname{Id}_{R^{x}}\left(S_{A R^{x}}^{x}\right)\right)\right) .
\end{aligned}
$$

Покажем, что функция $S_{A} \mapsto H_{\mathrm{q}}\left(S_{A}\right)-H_{\mathrm{cq}}\left(\mathscr{M} \otimes \operatorname{Id}_{R}\left(S_{A R}\right)\right)$, где $S_{A R}$ - очищение $S_{A}$, является вогнутой. Используя (1) и тот факт, что энтропии фон Неймана частичных состояний чистого состояния $\left(\operatorname{Tr} V(\omega) S_{A} V(\omega)^{*}\right)^{-1}\left(V(\omega) \otimes I_{R}\right) S_{A R}\left(V(\omega)^{*} \otimes I_{R}\right)$ равны, заметим, что

$$
H_{\mathrm{cq}}\left(\mathscr{M} \otimes \operatorname{Id}_{R} S_{A R}\right)=\int_{\Omega} H_{\mathrm{q}}\left(V(\omega) S_{A} V(\omega)^{*}\right) \mu(d \omega) .
$$

Условие вогнутости равносильно следующему:

$-\sum_{x} \pi_{x} H_{\mathrm{q}}\left(S_{A}^{x} \| \bar{S}_{A}\right) \leqslant-\sum_{x} \pi_{x} \int_{\Omega} H_{\mathrm{q}}\left(V(\omega) S_{A}^{x} V(\omega)^{*} \| V(\omega) \bar{S}_{A} V(\omega)^{*}\right) \mu(d \omega)$,

а для каждого слагаемого неравенство «ミ» имеет место в силу свойства монотонности относительной энтропии. 
Получаем, что

$$
\begin{aligned}
\sum_{x} \pi_{x}[ & \left.H_{\mathrm{q}}\left(S_{A}^{x}\right)-H_{\mathrm{cq}}\left(\mathscr{M} \otimes \operatorname{Id}_{R}^{x}\left(S_{A R^{x}}^{x}\right)\right)\right] \\
& \leqslant H_{\mathrm{q}}\left(\bar{S}_{A}\right)-\int_{\Omega} H_{\mathrm{q}}\left(V(\omega) \bar{S}_{A} V(\omega)^{*}\right) \mu(d \omega) .
\end{aligned}
$$

Таким образом, левая часть неравенства (30) не превышает

$$
H_{\mathrm{q}}\left(\bar{S}_{A}\right)-\int_{\Omega} p(\omega) H_{\mathrm{q}}(\widehat{S}(\omega)) \mu(d \omega)=\operatorname{ER}\left(\bar{S}_{A}, \mathfrak{M}\right) .
$$

(Здесь $p(\omega)=\operatorname{Tr} V(\omega) \bar{S}_{A} V(\omega)^{*}, \widehat{S}(\omega)=p(\omega)^{-1} \operatorname{Tr} V(\omega) \bar{S}_{A} V(\omega)^{*}$.)

Окончательно получаем, что

$$
\widetilde{C}_{\text {ea }}^{(1)}(\mathscr{M}, \mathscr{A}) \leqslant \sup _{S_{A}: \operatorname{Tr} S_{A} F \leqslant E} \operatorname{ER}\left(S_{A}, \mathfrak{M}\right),
$$

или, применяя рассуждения к $n$-кратному использованию канала,

$$
\widetilde{C}_{\text {ea }}^{(n)}\left(\mathscr{M}^{\otimes n}, \mathscr{A}^{\otimes n}\right) \leqslant \sup _{S_{A}^{(n)} \in \mathfrak{S}\left(\mathscr{H}_{A}^{\otimes n}\right): \operatorname{Tr} S_{A}^{(n)} F^{(n)} \leqslant n E} \operatorname{ER}\left(S_{A}^{(n)}, \mathfrak{M}^{\otimes n}\right) .
$$

Величина $\operatorname{ER}(S, \mathfrak{M})$ обладает свойством субаддитивности, т.е.

$$
\operatorname{ER}\left(S_{A B}, \mathfrak{M} \otimes \mathfrak{N}\right) \leqslant \operatorname{ER}\left(S_{A}, \mathfrak{M}\right)+\operatorname{ER}\left(S_{B}, \mathfrak{N}\right)
$$

для любого состояния $S_{A B} \in \mathfrak{S}\left(\mathscr{H}_{A} \otimes \mathscr{H}_{B}\right)$ с частичными состояниями $S_{A}, S_{B}$ и эффективных измерений $\mathfrak{M}, \mathfrak{N}[12$, теорема 2].

Рассмотрим величину

$$
\sup _{S_{A}^{(n)} \in \mathfrak{S}\left(\mathscr{H}_{A}^{\otimes n}\right): \operatorname{Tr} S_{A}^{(n)} F^{(n)} \leqslant n E} \operatorname{ER}\left(S_{A}^{(n)}, \mathfrak{M}^{\otimes n}\right) .
$$

Воспользовавшись субаддитивностью $\operatorname{ER}(S, \mathfrak{M})$ и обозначив частичные состояния $S_{A}^{(n)}$ через $S_{A}^{j}$, получим, что

$$
\begin{aligned}
S_{A}^{(n)} \in \mathfrak{S}\left(\mathscr{H}_{A}^{\otimes n}\right): \operatorname{Tr} S_{A}^{(n)} F^{(n)} \leqslant n E & \operatorname{ER}\left(S_{A}^{(n)}, \mathfrak{M}^{\otimes n}\right) \\
\leqslant & \sup _{S_{A}^{(n)} \in \mathfrak{S}\left(\mathscr{H}_{A}^{\otimes n}\right): \operatorname{Tr} S_{A}^{(n)} F^{(n)} \leqslant n E} \sum_{j=1}^{n} \operatorname{ER}\left(S_{A}^{j}, \mathfrak{M}\right) \\
\leqslant & \sin \left(\frac{1}{n} \sum_{j=1}^{n} S_{A}^{j}, \mathfrak{M}\right) \\
\leqslant & \sup _{S_{A}^{(n)} \in \mathfrak{S}\left(\mathscr{H}_{A}^{\otimes n}\right): \operatorname{Tr} S_{A}^{(n)} F^{(n)} \leqslant n E} \operatorname{ER}\left(S_{A}, \mathfrak{M}\right) .
\end{aligned}
$$

Здесь мы воспользовались тем, что из условия $\operatorname{Tr} S_{A}^{(n)} F^{(n)} \leqslant n E$ следует

$$
\operatorname{Tr} \frac{1}{n} \sum_{j=1}^{n} S_{A}^{j} F \leqslant E
$$


для частичных состояний $S_{A}^{j}$ состояния $S_{A}^{(n)}$. Тем самым неравенство (29) доказано.

Теперь докажем, что

$$
C_{\text {ea }}(\mathscr{M}, \mathscr{A}) \geqslant \sup _{S_{A}: \operatorname{Tr} S_{A} F \leqslant E} \operatorname{ER}\left(S_{A}, \mathfrak{M}\right) .
$$

Для доказательства понадобится определение сильно типичного проектора [5].

О п р е д е л е н и е 11 . Зафиксируем $\delta>0$ и рассмотрим оператор плотности $S_{d}$ ранга $d$, имеющий собственные числа $\lambda_{j}, j=1, \ldots, d$, и соответствующие собственные векторы $\left|e_{j}\right\rangle$. Тогда собственные числа оператора $S_{d}^{\otimes n}$ суть $\lambda_{J}=\lambda_{j_{1}} \cdots \lambda_{j_{n}}$, а собственные векторы $\left|e_{J}\right\rangle=\left|e_{j_{1}}\right\rangle \otimes \cdots \otimes\left|e_{j_{n}}\right\rangle$. Последовательность $J$ называется сильно типичной, если числа $N(j \mid J)$ появлений символов $j$ в последовательности $J$ удовлетворяют условию

$$
\left|\frac{N(j \mid J)}{n}-\lambda_{j}\right|<\delta, \quad j=1, \ldots, d,
$$

причем $N(j \mid J)=0$, если $\lambda_{j}=0$. Пусть $\widehat{T}_{d}^{n, \delta}$ обозначает совокупность всех сильно типичных последовательностей. Сильно типичный проекmор - это спектральный проектор оператора $S_{d}^{\otimes n}$, определяемый формулой

$$
\widehat{P}^{n, \delta}=\sum_{J \in \widehat{T}_{d}^{n, \delta}}\left|e_{J}\right\rangle\left\langle e_{J}\right|
$$

По закону больших чисел $\lim _{n \rightarrow \infty} P^{n}\left(\widehat{T}_{d}^{n, \delta}\right)=1$, где $P^{n}-$ распределение вероятностей, задаваемое собственными числами $\lambda_{J}$. Более того, для произвольной функции $g(j)$ и последовательности $J \in \widehat{T}^{n, \delta}$ справедливо неравенство

$$
\left|\frac{g\left(j_{1}\right)+\cdots+g\left(j_{n}\right)}{n}-\sum_{j=1}^{d} \lambda_{j} g(j)\right|<\delta d \max g .
$$

Рассмотрим канал $\mathscr{M}^{\otimes n}$, который задается семейством операторов

$$
M\left(\omega^{(n)}\right)=M\left(\omega_{1}\right) \otimes \cdots \otimes M\left(\omega_{n}\right)
$$

(или семейством $V\left(\omega^{(n)}\right)=V\left(\omega_{1}\right) \otimes \cdots \otimes V\left(\omega_{n}\right)$ ) и входное состояние $S \in \mathscr{A}$ со спектральным разложением $S=\sum_{j} \lambda_{j}\left|e_{j}\right\rangle\left\langle e_{j}\right|$, где $\left\{e_{j}\right\}_{j=1}^{\infty}$ - ортонормированный базис в пространстве $\mathscr{H}_{A}$. Рассмотрим отображение $S \mapsto \operatorname{Tr} S F$; поскольку оператор $F$ по предположению не пропорционален единичному, образ выпуклого множества всех операторов плотности при данном отображении содержит некоторый интервал. Следуя [5], 
предположим сначала, что $E$ не является минимальным собственным значением оператора $F$. Тогда найдутся вещественное число $E^{\prime}$ и оператор плотности $S \in \mathfrak{S}\left(\mathscr{H}_{A}\right)$ такие, что $\operatorname{Tr} S F \leqslant E^{\prime}<E$. Рассмотрим вспомогательный оператор плотности

$$
S_{d}=\sum_{j=1}^{d} \widetilde{\lambda}_{j}\left|e_{j}\right\rangle\left\langle e_{j}\right|, \quad \text { где } \widetilde{\lambda}_{j}=\left(\sum_{i=1}^{d} \lambda_{i}\right)^{-1} \lambda_{j},
$$

тогда $\left\|S_{d}-S\right\|_{1} \rightarrow 0$ при $d \rightarrow \infty$ и

$$
\operatorname{Tr} S_{d} F=\sum_{j=1}^{d} \widetilde{\lambda}_{j}\left\|\sqrt{F} e_{j}\right\|^{2}=E^{\prime}+\varepsilon_{d},
$$

где $\varepsilon_{d} \rightarrow 0$ при $d \rightarrow \infty$.

Рассмотрим сильно типичный проектор оператора $S_{d}^{\otimes n}$. Положим $k_{n, \delta}=\operatorname{dim} \widehat{P}^{n, \delta}=\left|\widehat{T}_{d}^{n, \delta}\right|, \bar{S}_{d}^{n, \delta}=\left(k_{n, \delta}\right)^{-1} \widehat{P}^{n, \delta}$ и заметим, что при достаточно больших $d$ можно выбрать $\delta_{0}$ так, что при $\delta<\delta_{0}$

$$
\operatorname{Tr} \bar{S}_{d}^{n, \delta} F^{(n)} \leqslant n E
$$

(более подробно см. [5]).

Обозначим $p^{n, \delta}\left(\omega^{(n)}\right)=\operatorname{Tr} M\left(\omega^{(n)}\right) \bar{S}_{d}^{n, \delta}, p(\omega)=\operatorname{Tr} M(\omega) S$ и покажем сначала, что

$$
\widetilde{C}_{e a}^{(n)}\left(\mathscr{M}^{\otimes n}, \mathscr{A}^{(n)}\right) \geqslant \operatorname{ER}\left(\bar{S}_{d}^{n, \delta}, \mathfrak{M}^{\otimes n}\right) .
$$

Заметим, что для измерительных каналов $\mathscr{M}$, удовлетворяющих (27), для любого входного состояния $S_{A}$ выполняется равенство

$$
\operatorname{ER}\left(S_{A}, \mathfrak{M}\right)=H_{\mathrm{q}}\left(S_{A}\right)+H_{\mathrm{c}}\left(\mathscr{M}\left(S_{A}\right)\right)-H_{\mathrm{cq}}\left(\mathscr{M} \otimes \operatorname{Id}_{R}\left(S_{A R}\right)\right),
$$

где $S_{A R}$ - очищение $S_{A}$.

Зададим конкретный протокол передачи классической информации с использованием сцепленности. Пусть классический сигнал принимает значения $(x, y), x, y=1, \ldots, k_{n, \delta}$ с равными вероятностями $\pi_{x, y}=1 / k_{n, \delta}^{2}$. В качестве сцепленного состояния возьмем

$$
S_{A B}=\left|\psi_{A B}\right\rangle\left\langle\psi_{A B}\right|, \quad \text { где }\left|\psi_{A B}\right\rangle=\frac{1}{\sqrt{k_{n, \delta}}} \sum_{J \in \widehat{T}_{d}^{n, \delta}}\left|e_{J}\right\rangle \otimes\left|e_{J}\right\rangle .
$$

Рассмотрим дискретную систему Вейля $\{W(x, y)\}$ (см. $[2$, раздел 8.4]) и зададим кодирования $\mathscr{E}_{A}^{x y}(\cdot)=W(x, y)(\cdot) W(x, y)^{*}$.

Тогда из определения $C_{\text {еа }}$ следует, что

$$
\begin{aligned}
C_{\mathrm{ea}}\left(\mathscr{M}^{\otimes n}, \mathscr{A}^{(n)}\right) \geqslant & H_{\mathrm{cq}}\left(\frac{1}{k_{n, \delta}^{2}} \sum_{x, y}\left(\mathscr{M} \otimes \operatorname{Id}_{B}\right)^{\otimes n}\left(S_{A B}^{x y}\right)\right) \\
& -\frac{1}{k_{n, \delta}^{2}} \sum_{x, y} H_{\mathrm{cq}}\left(\left(\mathscr{M} \otimes \operatorname{Id}_{B}\right)^{\otimes n}\left(S_{A B}^{x y}\right)\right)
\end{aligned}
$$


(здесь $\left.S_{A B}^{x y}=W(x, y) \otimes I_{B}^{\otimes n} S_{A B}\left(W(x, y) \otimes I_{B}^{\otimes n}\right)^{*}\right)$. Заметим, что при таком выборе кодирования ансамбль удовлетворяет ограничению на входе, поскольку

$$
\sum_{x, y} \pi_{x, y}\left(W(x, y) \otimes I_{B}^{\otimes n}\right) S_{A B}\left(W(x, y) \otimes I_{B}^{\otimes n}\right)^{*}=\bar{S}_{d}^{n, \delta} \otimes \bar{S}_{d}^{n, \delta}
$$

Тогда

$$
H_{\mathrm{cq}}\left(\frac{1}{k_{n, \delta}^{2}} \sum_{x, y}\left(\mathscr{M} \otimes \operatorname{Id}_{B}\right)^{\otimes n}\left(S_{A B}^{x y}\right)\right)=H_{\mathrm{c}}\left(\mathscr{M}^{\otimes n}\left(\bar{S}_{d}^{n, \delta}\right)\right)+H_{\mathrm{q}}\left(\bar{S}_{d}^{n, \delta}\right) .
$$

Поскольку состояние $S_{A B}^{x y}$ является очищением состояния $\bar{S}_{d}^{n, \delta}$, получаем, что правая часть (32) равна

$$
\begin{aligned}
\operatorname{ER}\left(\bar{S}_{d}^{n, \delta}, \mathfrak{M}^{\otimes n}\right) & =H_{\mathrm{q}}\left(\bar{S}_{d}^{n, \delta}\right)+H_{\mathrm{c}}\left(\mathscr{M}^{\otimes n}\left(\bar{S}_{d}^{n, \delta}\right)\right)-H_{\mathrm{cq}}\left(\left(\mathscr{M} \otimes \operatorname{Id}_{B}\right)^{\otimes n}\left(S_{A B}^{x y}\right)\right) \\
& =H_{\mathrm{q}}\left(\bar{S}_{d}^{n, \delta}\right)-\int_{\Omega} p^{n, \delta}(\omega) H_{\mathrm{q}}\left(\frac{V(\omega) \bar{S}_{d}^{n, \delta} V(\omega)^{*}}{p^{n, \delta}(\omega)}\right) \mu(d \omega)
\end{aligned}
$$

(здесь $\left.p^{n, \delta}(\omega)=\operatorname{Tr} V(\omega) \bar{S}_{d}^{n, \delta} V(\omega)^{*}\right)$, откуда следует неравенство (31).

Теперь обозначим $p(\omega)=\operatorname{Tr} V(\omega) S_{d} V(\omega)^{*}, p^{n, \delta}\left(\omega^{(n)}\right)=\operatorname{Tr} V\left(\omega^{(n)}\right) \times$ $\bar{S}_{d}^{n, \delta} V\left(\omega^{(n)}\right)^{*}$ и покажем, что

$$
\begin{aligned}
& \lim _{\delta \rightarrow 0} \lim _{n \rightarrow \infty} \frac{1}{n} \int_{\Omega^{\times n}} p^{n, \delta}\left(\omega^{(n)}\right) H_{\mathrm{q}}\left(\frac{V\left(\omega^{(n)}\right) \bar{S}_{d}^{n, \delta} V\left(\omega^{(n)}\right)^{*}}{p^{n, \delta}\left(\omega^{(n)}\right)}\right) \mu\left(d \omega^{(n)}\right) \\
&=\int_{\Omega} p(\omega) H_{\mathrm{q}}\left(\frac{V(\omega) S_{d} V(\omega)^{*}}{p(\omega)}\right) \mu(d \omega) .
\end{aligned}
$$

Отсюда будет следовать равенство

$$
\lim _{\delta \rightarrow 0} \lim _{n \rightarrow \infty} \frac{1}{n} \operatorname{ER}\left(\bar{S}_{d}^{n, \delta}, \mathfrak{M}^{\otimes n}\right)=\operatorname{ER}\left(S_{d}, \mathfrak{M}\right) .
$$

Рассмотрим разность

$$
\begin{aligned}
& n \int_{\Omega} p(\omega) H_{\mathrm{q}}\left(\frac{V(\omega) S_{d} V(\omega)^{*}}{p(\omega)}\right) \mu(d \omega) \\
& \quad-\int_{\Omega^{\times n}} p^{n, \delta}\left(\omega^{(n)}\right) H_{\mathrm{q}}\left(\frac{V\left(\omega^{(n)}\right) \bar{S}_{d}^{n, \delta} V\left(\omega^{(n)}\right)^{*}}{p^{n, \delta}\left(\omega^{(n)}\right)}\right) \mu\left(d \omega^{(n)}\right)
\end{aligned}
$$

и заметим, что поскольку из неотрицательности $E R\left(S_{d}, \mathfrak{M}\right)$ следует, что

$$
\int_{\Omega} p(\omega) H_{\mathrm{q}}\left(\frac{V(\omega) S_{d} V(\omega)^{*}}{p(\omega)}\right) \mu(d \omega) \leqslant H_{q}\left(S_{d}\right)<\infty,
$$

то разность (33) корректно определена. 
Прибавим и вычтем в (33) слагаемое

$$
\begin{gathered}
-\int_{\Omega^{\times n}} p^{n, \delta}\left(\omega^{(n)}\right) \operatorname{Tr} \frac{V\left(\omega^{(n)}\right) \bar{S}_{d}^{n, \delta} V\left(\omega^{(n)}\right)^{*}}{p^{n, \delta}\left(\omega^{(n)}\right)} \log \frac{V\left(\omega^{(n)}\right) S_{d}^{\otimes n} V\left(\omega^{(n)}\right)^{*}}{p\left(\omega^{(n)}\right)} \mu\left(d \omega^{(n)}\right) \\
=-\frac{1}{k_{n, \delta}} \sum_{J \in \widehat{T}^{n, \delta}} \int_{\Omega^{\times n}} \operatorname{Tr} V\left(\omega_{1}\right)\left|e_{j_{1}}\right\rangle\left\langle e_{j_{1}}\left|V\left(\omega_{1}\right)^{*} \otimes \cdots \otimes V\left(\omega_{n}\right)\right| e_{j_{n}}\right\rangle\left\langle e_{j_{n}}\right| V\left(\omega_{n}\right)^{*} \\
\times\left(\log \frac{V\left(\omega_{1}\right) S_{d} V\left(\omega_{1}\right)^{*}}{p\left(\omega_{1}\right)} \otimes I \otimes \cdots \otimes I+\cdots\right. \\
\left.+I \otimes \cdots \otimes I \otimes \log \frac{V\left(\omega_{n}\right) S_{d} V\left(\omega_{n}\right)^{*}}{p\left(\omega^{(n)}\right)}\right) \mu\left(d \omega^{(n)}\right) \\
=\frac{1}{k_{n, \delta}} \sum_{J \in \widehat{T}^{n, \delta}}\left(g\left(j_{1}\right)+\cdots+g\left(j_{n}\right)\right),
\end{gathered}
$$

где

$$
g(j)=-\int_{\Omega} \operatorname{Tr} V(\omega)\left|e_{j}\right\rangle\left\langle e_{j}\right| V(\omega)^{*} \log \frac{V(\omega) S_{d} V(\omega)^{*}}{p(\omega)} \mu(d \omega) .
$$

(Здесь используется обобщение свойства логарифма $\log (A \otimes B)=\log A \otimes$ $I+I \otimes \log B$, где в случае вырожденных $A$ или $B$ рассматриваются сужения на подпространства $\operatorname{supp} A$ и $\operatorname{supp} B$.) Заметим, что сумма (35) принимает только конечные значения при условии (34).

Получаем, что разность (33) равна

$$
\begin{aligned}
\int_{\Omega^{\times n}} p^{n, \delta} & \left(\omega^{(n)}\right) H_{q}\left(\frac{V\left(\omega^{(n)}\right) \bar{S}_{d}^{n, \delta} V\left(\omega^{(n)}\right)^{*}}{p^{n, \delta}\left(\omega^{(n)}\right)} \| \frac{V\left(\omega^{(n)}\right) S_{d}^{\otimes n} V\left(\omega^{(n)}\right)^{*}}{p\left(\omega^{(n)}\right)}\right) \mu\left(d \omega^{(n)}\right) \\
& +\frac{1}{k_{n, \delta}} \sum_{J \in \widehat{T}^{n, \delta}}\left(g\left(j_{1}\right)+\cdots+g\left(j_{n}\right)\right) \\
& -n \int_{\Omega} p_{\omega} \operatorname{Tr} \frac{V(\omega) S_{d} V(\omega)^{*}}{p(\omega)} \log \frac{V(\omega) S_{d} V(\omega)^{*}}{p(\omega)} \mu(d \omega) .
\end{aligned}
$$

Используя определение относительной энтропии для гибридных систем и свойство монотонности относительной энтропии, получаем оценку сверху для первого слагаемого в виде

$$
H_{\mathrm{c}}\left(p\left(\omega^{(n)}\right) \| p\left(\omega_{1}\right) \cdots p\left(\omega_{n}\right)\right)+H_{\mathrm{q}}\left(\bar{S}_{d}^{n, \delta} \| S_{d}^{\otimes n}\right) \leqslant 2 H_{\mathrm{q}}\left(\bar{S}_{d}^{n, \delta} \| S_{d}^{\otimes n}\right),
$$

что стремится к нулю при $n \rightarrow \infty, \delta \rightarrow 0$ в силу свойств сильно типичного проектора, как показано в $[2$, раздел 8.4 .2$]$. Для остальных слагаемых в (36) имеет место оценка

$$
\frac{n}{k_{n, \delta}} \sum_{J \in \widehat{T}_{d}^{n, \delta}}\left|\frac{g\left(j_{1}\right)+\cdots+g\left(j_{n}\right)}{n}-\sum_{j=1}^{d} \widetilde{\lambda}_{j} g(j)\right|<n d \delta \max g .
$$


Это доказывает, что $C_{\text {еа }}(\mathscr{M}) \geqslant \mathrm{ER}\left(S_{d}, \mathfrak{M}\right)$ для произвольного входного состояния $S_{d}$, удовлетворяющего условию $\operatorname{Tr} S_{d} F \leqslant E$. Устремляя $d$ к бесконечности, имеем

$$
\lim _{d \rightarrow \infty} \operatorname{ER}\left(S_{d}, \mathfrak{M}\right) \geqslant \operatorname{ER}(S, \mathfrak{M})
$$

так как по теореме 2 из [12] для любой последовательности состояний $S_{n}$, сходящихся к $S_{0}$, и любого эффективного измерения $\mathfrak{M}$ из условия

$$
\lim _{n \rightarrow \infty} H_{\mathrm{q}}\left(S_{n}\right)=H_{\mathrm{q}}\left(S_{0}\right)<\infty
$$

следует

$$
\lim _{n \rightarrow \infty} \operatorname{ER}\left(S_{n}, \mathfrak{M}\right)=\operatorname{ER}\left(S_{0}, \mathfrak{M}\right)<\infty,
$$

а последовательность состояний $S_{d}$ такова, что условие $(37)$ выполняется $([13$, лемма 4$])$.

Получаем

$$
C_{\text {ea }}(\mathscr{M}, \mathscr{A}) \geqslant \operatorname{ER}(S, \mathfrak{M})
$$

для произвольного входного состояния $S$ такого, что $\operatorname{Tr} S F<E$. Для операторов $S$ с условием $\operatorname{Tr} S F=E$ можно дополнительно рассмотреть операторы $S_{\epsilon}=(1-\epsilon) S+\epsilon|e\rangle\langle e|$, где $\langle e|F| e\rangle<E$, тогда $\operatorname{Tr} S_{\epsilon} F<E$. Устремляя $\epsilon$ к нулю и пользуясь полунепрерывностью снизу $\operatorname{ER}(S, \mathfrak{M})$, распространяем неравенство (38) на входные состояния с условием $\operatorname{Tr} S F \leqslant E$.

Если $E$ - минимальное собственное значение $F$, то благодаря условию (19) из ограничения $\operatorname{Tr} S F \leqslant E$ вытекает, что носитель $S$ конечномерен, а значит, можно принять $S_{d}=S$ и повторить предыдущие рассуждения.

Таким образом, прямое утверждение теоремы кодирования также доказано.

\section{СПИСОК ЛИТЕРАТУРЫ}

1. Нильсен М. А., Чанг И. Л. Квантовые вычисления и квантовая информация. М.: Мир, 2006, 822 с.

2. Холево А. С. Квантовые системы, каналы, информация. М.: МЦНМО, 2010, 327 с.

3. Bennett C.H., Shor P.W., Smolin J.A., Thaplyal A.V. Entanglement-assisted capacity of a quantum channel and the reverse Shannon theorem. - IEEE Trans. Inform. Theory, 2002, v. 48, № 10, p. 2637-2655.

4. Холево А.C. Информационная емкость квантовой наблюдаемой. - Проблемы передачи информации, 2012, т. 48, № 1, с. 3-13.

5. Холево А. С. Классические пропускные способности квантового канала с ограничением на входе. - Теория вероятн. и ее примен., 2003, т. 48, в. 2, с. 359-374.

6. Рид М., Саймон Б. Методы современной математической физики, т. 1. М.: Мир, $1985,357 \mathrm{c}$.

7. Barchielli A., Lupieri G. Instruments and channels in quantum information theory. Optics and Spectroscopy, 2005, v. 99, p. 425-432. 
8. Barchielli A., Lupieri G. Instruments and mutual entropies in quantum information. - Banach Center Publ., 2006, v. 73, p. 65-80.

9. Кузнеиова A. A. Условная энтропия для бесконечномерных квантовых систем. Теория вероятн. и ее примен., 2010, т. 55, в. 4, с. 782-790.

10. Wehrl A. General properties of entropy. - Rev. Modern Phys., 1978. v. 50, p. 221260.

11. Cover T. M., Thomas J. A. Elements of Information Theory. New York: Wiley, 1991, $542 \mathrm{p}$.

12. Shirokov M. E. Entropy reduction of quantum measurements. - J. Math. Phys., 2011, v. 52, № 5, paper № 052202, 18 p.

13. Lindblad $G$. Expectations and entropy inequalities for finite quantum systems. Comm. Math. Phys., 1974, v. 39, p. 111-119.

Поступила в редакцию 